\title{
Möglichkeiten der Nutzung von Media-Analyse- Radiodaten für Sekundäranalysen von 1972 bis heute
}

\author{
Jörg Hagenah
}

Seitvielen Jabren stehen die Daten der Media-Analyse fürwissenschaftliche Sekundäranalysen bereit. Bis 2002 wurden sie allerdings nur selten von Kommunikationswissenschaftlern genutzt. Dies hat sich mit der technischen Aufbereitung der Daten in das SPSS-Format ein wenig geändert, doch lässt sich bis dato nicht von einer problemlosen Datennutzung sprechen. Insbesondere eine wünschenswerte longitudinale Nutzung ist aufgrund der Unübersichtlichkeit der Datenmengen kaum von einzelnen Wissenschaftlern innerhalb des Arbeitsalltags zu realisieren. Daber wurde eine Dokumentenanalyse der Fragebögen und Codepläne für den Kernbereich der senderspezifischen Radionutzung durchgeführt, so dass nun das 1987 eingefübrte aktuelle Abfragemodell dem davor liegenden gegenübergestellt werden kann: Problemlos lassen sich die Abfrageblöcke zu General-und Zeitfilter längsschnittlich nutzen; methodisch problematisch zeigt sich jedoch die longitudinale Nutzbarkeit von Frequenzabfrage und Tagesablauf auf der einen sowie der abgeleiteten Nutzungswabrscheinlichkeiten, Kontaktsummen und Varianzen auf der anderen Seite.

Schlagwörter: Hörfunk, Sekundäranalyse, Longitudinalforschung, Mediennutzung, Methoden

Der Ruf nach Longitudinalforschung gehört nach Kiefer (1998: 27) in der Kommunikationswissenschaft genauso zur Tradition (Kaase \& Langenbucher 1986; Murray 1991) wie der Mangel an Forschung dieser Art. Die Kommunikationswissenschaft habe so gut wie keine Erfahrung mit Langzeitforschung ${ }^{1}$ (Kiefer 1999: 254). Allerdings sei das nicht Folge fehlender Daten (Kiefer 1998: 28), denn für Langzeitanalysen bietet nach Lauf (2006: 69) vor allem die Media-Analyse ${ }^{2}$ (MA) ${ }^{3}$ eine einzigartige Datenquelle, „die

1 Basierend auf der Studie Massenkommunikation wurden jedoch regelmäßig Zeitreihen (u. a. Ridder \& Engel 2005; Van Eimeren \& Ridder 2001; Berg \& Ridder 2002; Kiefer 1998; Berg \& Kiefer 1992) und Kohortenanalysen (Engel \& Best 2001; Peiser 1996) publiziert, die allerdings im besten Falle Lücken von vier Jahren aufweisen.

2 Hagenah \& Akinci 2003a: „Der Auftraggeber der MA ist die Arbeitsgemeinschaft Media-Analyse (AG.MA), ein Zusammenschluss von mehr als 250 Unternehmen der deutschen Werbewirtschaft, die für die Erhebungen jährlich mehr als 8 Millionen Euro ausgeben. Zu den Mitgliedern zählen Werbungtreibende, Werbe- und Media-Agenturen, Pressemedien, elektronische Medien und Mitglieder der Gruppe Plakat. Die Datensätze der MA gelten als sog. Werbewährung insbesondere für Radiosender und Pressemedien. Sie dienen allen Programmanbietern und auch der Werbewirtschaft als zentrale Informationsquelle und beinhalten die aktuellen Nutzungsdaten für die Mediengattungen Radio, Fernsehen, Zeitungen/Zeitschriften, Kino, Lesezirkel, Konpress und zukünftig auch für Plakate und Onlineangebote. Die jeweils aktuellen Daten aus dem zurückliegenden Erhebungsjahr stehen zunächst nur den Mitgliedern der AG.MA für planungsrelevante Entscheidungen zur Verfügung. Nach einer gewissen Karenzzeit sind die gesamten Datensätze stets an das Zentralarchiv für Empirische Sozialforschung (ZA) zur wissenschaftlichen Verwendung weitergegeben worden. “ Zurzeit können die aufbereiteten Datensätze und Datensatzdokumentationen kostenlos beim Medienwissenschaftlichen Lehr- und Forschungszentrum geordert werden.

3 Die Arbeitgemeinschaft Media-Analyse schreibt ma mittlerweile klein. In diesem Beitrag wird 
- unverständlich genug - von der Kommunikationswissenschaft bis heute weitgehend ignoriert wurde“. Dies zeigte auch eine von Hasebrink 2002 durchgeführte Bestandsaufnahme zur Praxis von Sekundäranalysen in der Kommunikationswissenschaft. Die MA wurde bis dato nur selten von Kommunikationswissenschaftlern sekundär analysiert (Kubitschke \& Trebbe 1992; Weiß \& Hasebrink 1995, 1997; Schönbach, Lauf, Stürzebecher \& Peiser 1997; Lauf 1999). Längere MA-Zeitreihen unter Ausnutzung der vollen Spanne wurden noch nicht erstellt, obwohl dies der einzige Datensatz ist, der durchgängig jährlich erhobene Kennwerte zur Mediennutzung zu bieten hat.

Die geringe Nutzung lässt sich vor allem mit der binären Datenstruktur der ins ZA gelieferten Originaldateien erklären, die nicht mit SPSS kompatibel waren, so dass vor jeder Nutzung aufwändige Konvertierungsarbeiten notwendig waren (Hagenah, Meulemann \& Akinci 2006). Die technischen Probleme wurden in den Jahren 2003 bis 2005 vom Medienwissenschaftlichen Lehr- und Forschungszentrum der Universität zu Köln (MLFZ) gelöst. In Zusammenarbeit mit dem Zentralarchiv für empirische Sozialforschung Köln wurden die 184 binären MA-Originaldateien der Jahre 1972 bis 2003 und die 14 Dateien der Vorgängerstudie Leser-Analyse (LA) in das gängige SPSS-Format konvertiert. Jährlich werden seitdem - die jeweils etwa ein Jahr alten - aktuellsten Datensätze konvertiert. Alle Datensätze können kostenlos beim MLFZ geordert werden, zudem erfolgt eine fachliche Beratung ${ }^{4}$.

Die Größe der MA bringt jedoch darüber hinaus Probleme der Übersichtlichkeit mit sich: Von Anfang an setzte sich der Datenbestand der Media-Analyse aus einer Vielzahl von Variablen zusammen. Schon im Vorwort zum MA 75 Berichtsband (1975: 4) lassen sich ein paar bezeichnende Sätze finden:

„Die zweite Ebene der Schwierigkeiten betrifft die Datenfülle. [...] Die Frage bleibt offen, ob eine leichter zugängliche Form der Berichterstattung überhaupt möglich erscheint. Letztlich ist das wirkliche Kernstück der Berichterstattung der Datensatz. Der Umgang mit diesem jedoch variiert und muß erlernt werden."

Da, wie Scheler (1979: 1369) Ende der 1970er Jahre über die MA schrieb, „die möglichen Datenverknüpfungen und Auswertungen so zahlreich sind, dass die Erde längst nicht mehr stehen würde, wollte man alle bilden", wurden die Erhebungen und deren Variablen vom MLFZ (Hagenah \& Akinci, 2003b) in der MA-Datensynopse „madatsyn 1.0" inventarisiert. Sie enthält auf 33.483 Zeilen alle der ca. 32.000 mindestens einmal erhobenen Einzelvariablen von 1972 bis 2000 mit ihren Erhebungszeitpunkten und Speicherorten in einer EXCEL-Tabelle.

Technische Aufbereitung und Datenservice haben die Publikationstätigkeiten seit 2005 erleichtert, wie insbesondere in einem Herausgeberband von Hagenah und Meulemann ersichtlich ist, in dem ausschließlich MA-Sekundäranalysen zusammengestellt sind (Hagenah \& Meulemann 2006). Der Band enthält neben sozialwissenschaftlichen Untersuchungen (Fachinger 2006; Wahl 2006; Risel 2006) kommunikationswissen-

MA einheitlich groß geschrieben, da dies - über die Zeit gesehen - auch in den meisten Erhebungen und Publikationen so gehandhabt wurde.

4 Als Anreiz für die Analyse wurde vom MLFZ eine CD mit den Daten von 1975 bis 2000 in Fünfjahresschritten erstellt und interessierten Wissenschaftlern kostenlos zur Verfügung gestellt. Zusätzlich wurden im Bedarfsfall individuelle Datensätze zusammengestellt. Fragebögen können von der MLFZ-Homepage www.wiso.uni-koeln.de/medien/frei herunter geladen werden. Analyseleitfäden wurden erstellt (Hagenah 2003a, 2003b, Hagenah 2004a; Akinci 2004), Nutzer wurden fachlich beraten und ein Workshop wurde ausgerichtet (Hagenah 2004b). 
schaftliche Beiträge von erfahrenen MA-Autoren, die zu neuen Studien angeregt wurden (Müller \& Mai 2006; Lauf 2006; Best \& Hagenah 2006; Hagenah \& Schliermann 2006) und Beiträge von Personen, die erstmalig MA-Untersuchungen publizierten (Akinci 2006; Seufert \& Suckfüll 2006; Gonser \& Scherer 2006; Doh \& Kaspar 2006). Darüber hinaus wurden erste Diplom- und Magisterarbeiten auf Basis der MA-Daten geschrieben (z. B. Risel 2005; Frisch 2005; Ehrenberg 2005).

Dennoch lässt sich die Arbeit mit MA-Daten nicht als unproblematisch und unkompliziert bezeichnen. Erstens ist die Übersichtstabelle madatsyn1.0 so groß, dass es immer noch einer großen Einarbeitungszeit bedarf, um tatsächlich einen Überblick über die über 30.000 Variablen zu bekommen. Zweitens erspart das Servicetool nicht den Blick in zumindest einen der unübersichtlichen Codepläne, da die Dateien noch weitestgehend ungelabelt sind. Drittens ist insbesondere der longitudinale Gebrauch der Daten mühsam, da nicht nur aus vielen Codeplänen die Variablennummern recherchiert werden müssen; vielmehr muss auch noch ein Vergleich der Variablencodierungen getätigt und ggf. ein Umcodierungsplan entwickelt werden. Folge dieses weiteren Aufbereitungsbedarfs ist, dass einerseits einige Datenbesitzer ${ }^{5}$ die Daten vermutlich eher horten als nutzen; andererseits beschränkt sich die bisherige Nutzung vor allem auf wenige - überwiegend aktuellere - Datensätze. Longitudinale Untersuchungen sind eher selten und behandeln ausschließlich die jüngere Rezeptionsgeschichte, weiter zurück reichende historische Ansätze wurden bisher nicht verfolgt.

Ziel dieses Beitrags ist es, für die Abfrage der Hörfunknutzung einen detaillierten Überblick darüber zu verschaffen, welche Variablen auf welche Weise wann erhoben wurden. Dies erscheint aus drei Gründen sinnvoll. Erstens können mit Hilfe der Untersuchung unterschiedliche Forscher aus divergierenden Perspektiven gezielt MAZeitreihen erstellen. Zweitens soll somit die methodische Herangehensweise bei der Erstellung von MA-Zeitreihen zur Diskussion gestellt werden, um nicht ähnliche Vergleichbarkeitsprobleme zu bekommen wie die Langzeitstudie Massenkommunikation, deren Berichtsbände laut Lauf und Peiser (1999: 240ff.) erhebliche Mängel aufweisen. Drittens wird die methodische Abfragepraxis der Mediaforschung weiter für die Scientific Community offen gelegt als bisher geschehen und kann als Beispiel oder Vorlage für universitäre Forschungsaktivitäten dienen ${ }^{6}$ oder als Grundlage für eine kritische Betrachtung der Mediaforschungspraxis fungieren.

Als Informationsbasis für eine Dokumentenanalyse dienen die MA-Codepläne und -Fragebögen der Jahre 1972 bis 2000. In einem vorgelagerten Schritt wird die Erhebungsstruktur dargestellt, um anhand existierender Umbrüche gezielt mögliche Veränderungen in der Variablenstruktur vermuten zu können.

\section{Erhebungs- und Variablenstruktur der Media-Analysen}

Erhebungsform, Erhebungsdichte und erhobene Medienarten der LA und der MA sind in Tabelle 1 mit ihren Wandlungen dargestellt.

Von 1954 bis 1958 wurde die Nutzung der Pressemedien in den LA alle zwei Jahre und dann bis 1971 jährlich erhoben. Seit 1972 wird in den MA auch die Nutzung der elektronischen Medien Radio und Fernsehen erhoben. Die Medienarten - Radio, Fern-

5 Als Datenbesitzer werden diejenigen Personen bezeichnet, die eine Daten-CD beim Medienwissenschaftlichen Lehr- und Forschungszentrum geordert haben.

6 Durch die Verwendung von MA-Abfrageblöcken könnten universitäre Studien ggf. nachträglich validiert werden. 
sehen, Zeitungen, Zeitschriften und Kino - werden seit 1987 nicht mehr gemeinsam abgefragt, sondern getrennt für Pressemedien (MA PM, Zeitungen und Zeitschriften) und elektronische Medien (MA EM, Radio und Fernsehen). Mit der MA 1997 wurden Daten zur Fernsehnutzung nicht mehr senderspezifisch erhoben, und der Schwerpunkt der MA EM liegt auf der Radionutzung, so dass im Jahr 2000 die MA-EM in MA Radio umbenannt wurden. Seit 1997 wird die MA PM, seit 2000 auch die MA Radio alle halbe Jahre erhoben.

Die Erhebungsform für die Messung der Radionutzung war von 1972 bis 1999 das persönlich-mündliche Interview ( $\mathrm{ADM}^{7}$-Stichprobenmodell, siehe Hoffmeyer-Zlotnik 1997; Behrens \& Löffler 1999). Seit 2000 wird die MA Radio telefonisch durch Computer-Assisted Telephone Interviews (CATI) erhoben (Müller \& Mai 2006: 23ff.; Hoffmann \& Müller 2003; Gabler \& Häder 1997): Heute werden zu jedem Zeitpunkt ca. 60.000 Personen telefonisch von mehreren Marktforschungsinstituten befragt.

Tabelle 1: Chronologie der Leser-Analysen (LA) und der Media-Analysen (MA)

\begin{tabular}{|c|c|c|c|c|}
\hline Jahre & Studie & Erhebungsform & $\begin{array}{l}\text { Erhebungs- } \\
\text { dichte }\end{array}$ & $\begin{array}{l}\text { Erhobene } \\
\text { Medienarten }\end{array}$ \\
\hline $1954-58 \%$ & LA & persönlich & zweijährlich & Presse \\
\hline $1960-71$ & & & jährlich & $\begin{array}{l}\text { Presse, Radio, } \\
\text { TV }\end{array}$ \\
\hline $1972-86$ & MA & & & \\
\hline $1987-96$ & $\begin{array}{l}\text { MA PM } \\
\text { MA EM }\end{array}$ & & & $\begin{array}{l}\text { Presse } \\
\text { Radio/TV }\end{array}$ \\
\hline 1997 - 99 & $\begin{array}{l}\text { MA PM } \\
\text { MA Radio }\end{array}$ & & $\begin{array}{l}\text { halbjährlich*** } \\
\text { jährlich*** }\end{array}$ & Presse \\
\hline Seit 2000 & $\begin{array}{l}\text { MA PM } \\
\text { MA Radio }\end{array}$ & $\begin{array}{l}\text { persönlich } * * \% \\
\text { telefonisch }\end{array}$ & $\begin{array}{l}\text { halbjährlich } \\
\text { halbjährlich } \% * \% * \%\end{array}$ & Radio \\
\hline
\end{tabular}

Quellen: Hagenah \& Meulemann (2006: 11); Meulemann, Hagenah \& Akinci (2005: 54)

PM = Pressemedien Tranche (Zeitungen, Zeitschriften); EM = Elektronische Medien Tranche (Radio, TV)

* Die LA 1958 ist - laut Auskunft der AG.MA - „verschollen“.

** 1998 wurde die MA Radio halbjährlich und die MA PM nur einmalig durchgeführt.

** Seit der 2. Erhebungswelle PM 2004 wird eine 10\%-Substichprobe mit der CASI-Methode (Computer-Assisted Self-administered Interview) befragt (www.agma-mmc.de, 2005). Zukünftig soll der Anteil sukzessive gesteigert werden.

**** Im Jahr 2000 wurde nur eine MA Radio erhoben.

Den größten Einfluss auf die Variablenstruktur hatte - wie im Folgenden auch ausführlicher gezeigt wird - die 1987 erfolgte Umstellung der Single-Source-Studie ${ }^{8}$ auf eine nach Medienart getrennte Abfrage, die zu der Einführung eines neuen (immer noch aktuellen) Abfragemodells geführt hat. Dies betrifft vor allem die originären, weniger die abgeleiteten und die interview-unabhängigen Variablen. Auf die beiden erstgenannten Variablenkomplexe wird ausführlich eingegangen. Die Letztgenannten sind beispielsweise Gemeindekoordinaten und -größen, die nicht erfragt wurden, sondern bei der Befragungsvorbereitung ermittelt wurden.

$7 \quad \mathrm{ADM}=$ Arbeitskreis Deutscher Markt- und Sozialforschungsinstitute.

8 Insbesondere in den 1970er-Jahren wurde synonym auch der Begriff Multi-Media-Analyse verwendet. 
Während sich die originären Fragebogeninformationen auf die konkrete Auflistung der abgefragten Antworten beschränken, enthalten die abgeleiteten Teile neben den die Auswertungspraxis erleichternden Summen (u. a. auf Basis der Ursprungsdaten) nach bestimmten Konventionen neu berechnete Kennziffern (z. B. Nutzungswahrscheinlichkeiten). Insbesondere der zweite Teil bedarf einer genaueren Erklärung, die in Abschnitt 3 geliefert wird. Vorher werden in Kapitel 2 die originären Daten näher beleuchtet.

\section{Originäre Variablen: Sender-Abfragemodelle von 1972 bis heute}

Kernbereiche der Media-Analysen sind die Abfragen zur senderspezifischen Hörfunknutzung. In der Zeit von 1972 bis heute wurden zwei unterschiedliche Modelle verwendet, die im Folgenden beschrieben und miteinander verglichen werden.

Die Untersuchungsanlage der aktuellen MA Radio bezüglich der Nutzung spezifischer Radiosender sieht nach Müller und Mai (2006; Mai 2003) folgendermaßen aus: Dem Generalfilter folgen der Zeitfilter, eine Frequenzabfrage und die Fragen zum Tagesablauf (Abbildung 1). Den Fragebögen und Codeplänen 'ässt sich entnehmen, dass diese vier Schritte seit 1987 in identischer Form eingesetzt werden.

Mit Hilfe der Dokumentenanalyse lässt sich das ältere Abfragemodell der Zeit von 1972 bis 1986 rekonstruieren. Es ähnelt dem aktuellen Modell und hatte dasselbe Grundprinzip, dennoch lassen sich neben Gemeinsamkeiten auch ein paar Unterschiede konstatieren. Die ersten beiden Schritte sind identisch, dem Generalfilter folgt der Zeit-

\section{Abbildung 1: Das Modell der Abfrage der MA Radio (eigene Darstellung in Anlebnung an Mai 2003: 13)}

\section{Das Abfragemodell der MA Radio}

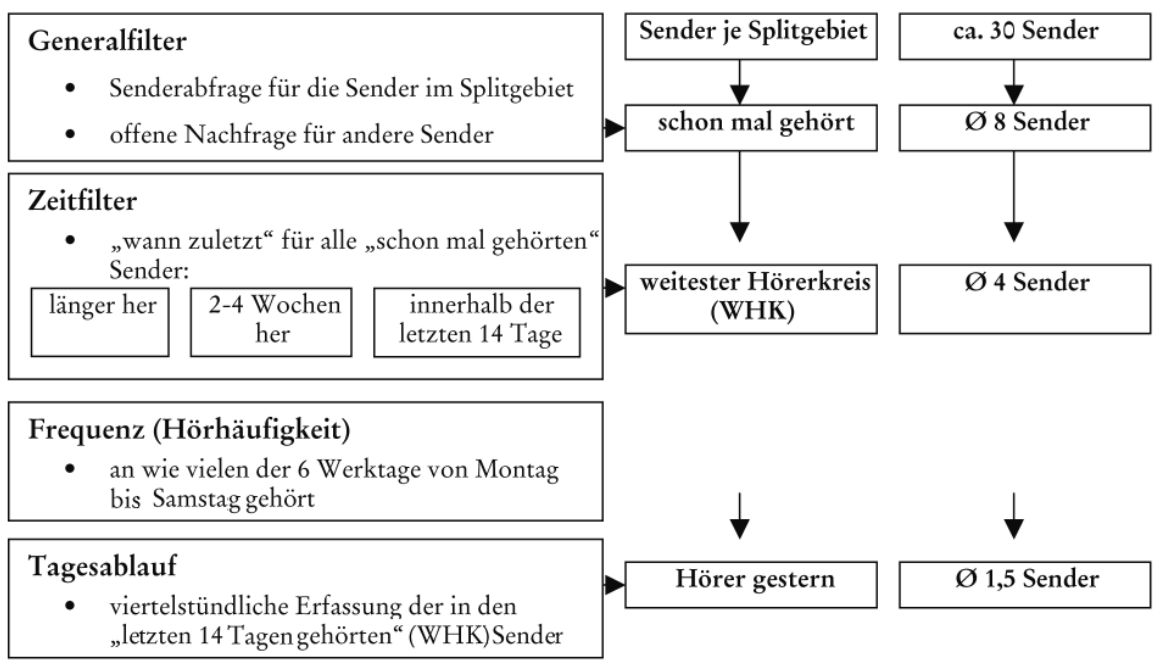

9 Alle Seitenangaben zu Fragebögen und Codeplänen beziehen sich auf die Seitenangaben im dazugehörigen PDF-Dokument. 
filter. Die Frequenzfrage (Hörhäufigkeit) wurde früher jedoch detaillierter erfasst und die Tagesablauffrage war gröber.

Leider muss für alle Radiovariablen des älteren Modells von 1972 bis 1975 - zumindest vorläufig ${ }^{10}$ - konstatiert werden, dass sie nur fragmentarisch vorhanden sind bzw. nicht vollständig dokumentiert vorliegen. Daher beschränken sich die folgenden Analysen auf die Erhebungen ab 1976.

Die einzelnen Kategorien der beiden Erhebungsmodelle werden in der genannten Reihenfolge näher vorgestellt. Zuerst werden die entsprechenden Variablenkomplexe des aktuellen Modells beschrieben, danach folgt ein Abgleich mit dem älteren Modell.

\subsection{Generalfilter}

Seit 1970 verwendeten die LA und deren Nachfolger die MA in allen Erhebungen einen Generalfilter, durch den sich die Befragten als Hörer bestimmter Sender „qualifizieren“ (vgl. Koschnick, 2004d). In diesem ersten Komplex wird danach gefragt, „welche Sender, welche Programme schon mal gehört wurden?“. Die konkreten Fragebogeninhalte sind in Abbildung $2 \mathrm{zu}$ finden. Fett gedruckt stehen die Anweisungen für den Interviewer, in Normalschrift die Passagen, die den Befragten vorgelesen werden. Um den Befragten die Erinnerung zu erleichtern und um den Befragungsprozess zu beschleunigen, werden so genannte "Titelkarten“ eingesetzt („gestützte Abfrage mit optischer Erinnerungshilfe“). Für jeden abgefragten Radiosender ${ }^{11}$ existiert eine Karte, die den Sendernamen (zum Teil mit Slogan oder Sendefrequenzangabe, vgl. Abb. 2) enthält. Vor jeder Umfrage soll das so genannte „Kartenspiel“ vom Interviewer neu gemischt werden, um mögliche Reihenfolgeeffekte zu vermeiden. Die Befragten werden nun gebeten, die Karten zu sortieren und entsprechend der Antwortmöglichkeiten zwei „Häufchen“ zu bilden. Die Karten von dem Häufchen „von diesen Sendern, Programmen habe ich noch nie gehört“ werden sofort weggesteckt, diejenigen von dem Häufchen „von diesen Sendern, Programmen habe ich schon mal gehört“ werden im Fragebogen als solche angekreuzt. Zu diesen Radiosendern werden auch Folgefragen gestellt.

Codiert werden die daraus gewonnenen Informationen - wie in Abb. 2 zu sehen - zusammen mit den Informationen aus der nachfolgenden Zeitfilterfrage. Unter den in den Codeplänen genannten Feldnummern befinden sich in den Datensätzen unter dem Oberbegriff „Zeitfilter“ sowohl die Daten aus der Generalfilterfrage als auch die Informationen, die aus der im engeren Sinne Zeitfilter genannten Frage stammen. Die Ausprägungen 1 bis 3 bei der Zeitfilterkodierung können zusammengenommen auch als Antwortmöglichkeit „schon mal gehört“ = ja zur Generalfilterfrage gewertet werden. 6 $=$ noch nie gehört und $5=$ keine Angabe stammen direkt aus der Generalfilterfrage.

Aufgrund der veränderten Befragungssituation sieht die Abfrage zum Generalfilter bei den seit 2000 telefonisch durchgeführten Interviews etwas anders aus. Die optischen Erinnerungshilfen fallen weg. Stattdessen werden durch Vorlesen von Sendernamen und

10 Beispielsweise finden sich zur MA 1975 Informationen zur Generalfilterabfrage in den Fragebögen und Codeplänen, in dem vom ZA aufbereiteten Datensatz sind sie jedoch nicht enthalten. Das mag daran liegen, dass während der Umbauphase von der LA zur MA noch experimentiert wurde. Nach Scheler (1983: 387) seien mit der „MA 76 die wesentlichen Probleme des Ausbaus zur Multi-Media-Analyse gelöst" worden.

11 Abgefragt werden nur diejenigen Sender, die im Wohngebiet des Befragten theoretisch auch empfangen werden können. Bei der Nachfrage können aber auch andere Sender genannt werden. 
Abbildung 2: Generalfilterabfrage im persönlich-mündlichen Interview: Fragebogen MA_99_EM (ma_99_EM_frb.pdf: 5, 81) und Codeplan MA 1999 EM (ma_99_EM_cdb.pdf: 87) (eigene Darstellung)

4 INT: Radio-Karten aus dem Umschlag „Hörfunk“ herausnehmen.

Blätter $4 \mathrm{R}, 4 \mathrm{~L}$ aufschlagen.

Auf diesen Karten stehen die Namen von verschiedenen Rundfunksendern und Radioprogrammen. Welche Sender, welche Programme haben Sie schon mal gehört?

INT: Radio-Karten übergeben. Vom Befragten auf die Blätter $4 \mathrm{R}$ und $4 \mathrm{~L}$ sortieren lassen.

Antworten im Schema ankreuzen. Falls keinen Sender „schon mal gehört“, weiter mit Frage 7.

Bitten Sie den Befragten, die Nummern der Hörfunk-Karten vorzulesen, damit Sie diese rascher ankreuzen können.

\begin{tabular}{c|c} 
HO*T FM & HIT-Radio ANTENNE SACHSEN \\
Auf Frequenz & $94,0 \mathrm{MHz} / 93,4 \mathrm{MHz}$ \\
& $\begin{array}{c}96,5 \mathrm{MHz} / 97,3 \mathrm{MHz} \\
98,1 \mathrm{MHz}\end{array}$
\end{tabular}

Hörfunk

Zeitfilter 1 innerhalb der letzten 2 Wochen

$2 \quad 2$ bis 4 Wochen

3 länger her

$4 \quad$ keine Angabe (aus Zeitfilter)

5 keine Angabe (aus Generalfilter)

6 noch nie gehört

Slogans diejenigen Radiosender ermittelt, die der Befragte „schon mal gehört hat“ („gestützte Abfrage" = aided recall). Das Bundesgebiet wird dabei in ca. 140 Splitgebiete aufgeteilt, so dass die dort wohnenden Befragten nur nach den etwa 30 bis 35 im Split verbreiteten Sendern abgefragt werden (Mai, 2003). Um auch wirklich alle gehörten Sender zu erfassen, gibt es eine offene Abfrage nach weiteren Sendern (Hagenah \& Akinci, 2003a).

Das CATI-Frageprogramm (siehe Abb. 3) ermöglicht eine automatisierte Filterführung und somit eine schnellere Befragung. Außerdem sollten hierbei weniger Fehler bei der Dateneingabe erfolgen, da durch die elektronische Eingabe keine weitere Codierprozedur nötig ist. Dafür können vorherige Fehler später kaum noch ausgeglichen werden.

Aufgrund der veränderten Erhebungsform können die Ergebnisse im Vergleich zur persönlichen Befragung abweichen (Best \& Hagenah, 2006; Hagenah \& Best 2005).

Insgesamt 125 Einzelsender mit einem ausreichenden Hörerstamm ${ }^{12}$ wurden mindes-

12 Es lässt sich unterscheiden zwischen abgefragten und ausgewiesenen Sendern. Von den über 300 abgefragten Sendern werden nur diejenigen in den Datensätzen ausgewiesen, die laut MA- 
Abbildung 3: Generalfilterabfrage im telefonischen Interview: Fragebogen MA_2000_ EM II (ma_00_EM_II_frb.pdf: 6) (eigene Darstellung)

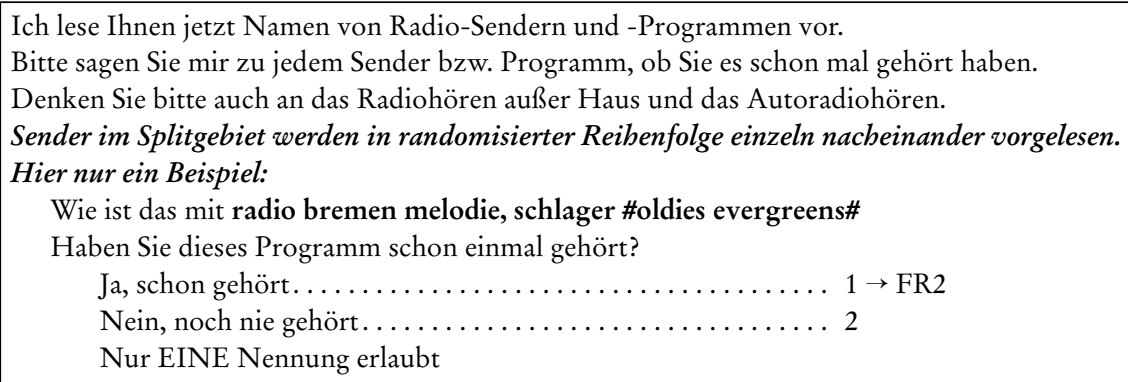

tens einmal zwischen 1972 und 2000 ausgewiesen. Die sieben ${ }^{13}$ öffentlich-rechtlichen Rundfunksender Sender Freies Berlin SFB $1(88,8)^{14}$, Bayerischer Rundfunk B1, Bayerischer Rundfunk B3, Hessischer Rundfunk HR1, Hessischer Rundfunk HR 3, WDR 1 Eins live und Hansawelle Radio Bremen sowie die in den 1990ern zu SWR 1 fusionierten Sender Südfunk1 Stuttgart SDR1 und SWF 1 (Radiodienst) Südwestfunk sind kontinuierlich seit Anfang der 1970er in den MA vertreten (vgl. madatsyn1.0, Zeilen 3170231826). 1988 wurde mit RSH Schleswig-Holstein der erste Privatsender ausgewiesen, seit Ende der 1980er/Anfang der 1990er ist die Anzahl der Untersuchungseinheiten enorm gestiegen (ausführlicher bei Hagenah \& Akinci, 2003b).

\subsection{Zeitfilter}

Für alle „schon mal gehörten Sender“ wird im so genannten Zeitfilter ermittelt, welche Sender in den letzten 14 Tagen gehört wurden (vgl. Mai, 2003: 12). Bei denjenigen Sendern, die vom Interviewten innerhalb der letzten zwei Wochen gehört worden sind, zählt der Befragte zum Weitesten Hörerkreis (WHK). Diese Zuordnung der Sender zum Weitesten Hörerkreis bildet die Voraussetzung für die sich anschließende Frequenzabfrage.

Auch bei der in Abbildung 4 dargestellten Zeitfilter-Frage dienten den persönlichmündlich Befragten die Senderkarten als optische Hilfe. Beim Telefoninterview werden die Sendernamen vorgelesen. Codiert wurden die Antworten wie in Abb. 4 dargestellt ${ }^{15}$.

Erhebung mindestens 351 Hörer haben (AG.MA 2004: 5).

13 Die Informationen stammen aus der Tabelle madatsyn1.0. Die entsprechenden Daten wurden den einzelnen Codeplänen entnommen und manuell eingegeben, d. h. dass auf Änderungen des Sendernamens u. U. nicht immer reagiert wurde. Entsprechende Sekundäranalysen müssten demnach eine diesbezügliche Senderrecherche umfassen.

14 Die Sendernamen haben sich über die Jahre verändert. Die in diesem Beitrag gewählten Namensversionen stammen aus der Tabelle madatsyn1.0.

15 Prinzipiell werden bei den neueren, telefonisch erhobenen Daten dieselben Kategorien verwendet, allerdings fällt eine der beiden „keine Angabe“-Kategorien weg, dadurch „verschiebt“ sich die „noch nie gehört“-Ausprägung (5 statt 6). Für vergleichende Untersuchungen müssen also Umkodierungen vorgenommen werden, die aber inhaltlich unbedenklich sind. 
Abbildung 4: Zeitfilterabfrage: Fragebogen MA_1999_EM (ma_99_EM_frb.pd:5) Fragebogen MA_2000_EM II (ma_00_EM_II_frb.pdf: 7) und Codeplan MA 1999 EM (ma_99_EM_cdb.pdf: 87) (eigene Darstellung)

5 INT: Frage 5 für alle lt. Frage 4 „schon mal gehörten“ Sender stellen. Blatt 5 aufschlagen.

Wann haben Sie zuletzt den gehört?

Hier habe ich eine Zeiteinteilung, die Ihnen vielleicht helfen kann, die richtige Antwort zu finden.

INT: Radio-Karten einzeln nacheinander auf Blatt 5 A vorlegen. Antworten im Schema ankreuzen.

Falls kein Sender „innerhalb der letzten 2 Wochen“ gehört wurde: weiter mit Frage 7.

CATI-Frageprogramm (c) MA 2000 Radio, 2. Welle

Nun folgt der Zeitfilter für alle Sender, die schon einmal gebört wurden.

Auch hier werden die Sender einzeln nacheinander vorgelesen. Ein Beispiel:

FR2 Wann haben Sie w d r zwei zuletzt gehört?

War das innerhalb der letzten 2 Wochen, innerhalb der letzten 2-4 Wochen oder ist es schon länger her?

Innerhalb der letzten 2 Wochen $\ldots \ldots \ldots \ldots \ldots \ldots \ldots(1)=$ WHK $\rightarrow$ FR3

Innerhalb der letzten $2-4$ Wochen ............... (2)

Nein, länger her....................... (3)

Nur EINE Nennung erlaubt!

Hörfunk

Zeitfilter 1 innerhalb der letzten 2 Wochen

$2 \quad 2$ bis 4 Wochen

3 länger her

$4 \quad$ keine Angabe (aus Zeitfilter)

5 keine Angabe (aus Generalfilter)

6 noch nie gehört

\subsection{Frequenz: Hörbäufigkeit (pro Zeitabschnitt) pro Sender}

In der dritten Stufe „Hörhäufigkeit pro Sender“ wird seit 1987 ermittelt, an wie vielen Werktagen von Montag bis Samstag jeder einzelne Sender gehört wird (siehe Abb. 6). Vor 1987 wurden die Informationen zur Hörhäufigkeit detaillierter pro (Tages-) Zeitabschnitt (bspw. 08:00 bis 09:00 Uhr) pro Sender erhoben. Auf die letztgenannte Erhebungsform und deren Kompatibilität zur aktuellen Abfrage wird nachher gesondert eingegangen.

Zur „Hörhäufigkeit pro Sender“ (Frequenz) werden seit 1987 alle Personen befragt, die dem „weitesten Hörerkreis“ (WHK) angehören und demnach den Einzelsender innerhalb der letzten zwei Wochen gehört haben. Wird ein Sender von einer Person an mehr als drei von sechs Tagen gehört, so wird er als Stammbörer bezeichnet. Die Personen, die einen Sender an drei oder weniger Tagen hören, gelten als Gelegenheitshörer. Beim telefonischen Interview werden die Frequenzangaben gleichermaßen erhoben (siehe Abb. 5), aber direkt eingegeben. 
Abbildung 5: Frequenzabfrage (Hörbäufigkeit pro Woche): Fragebogen MA_1999_ EM (ma_99_EM_frb.pdf: 5) und Fragebogen MA_2000_EM II (ma_00_ EM_II_frb.pdf: 7) (eigene Darstellung)

6 INT: Blatt 6 aufschlagen.

Frage 6 für alle Sender stellen, die lt. Frage 5 ,innerhalb der letzten 2 Wochen“ gehört wurden.

Wenn Sie an eine normale Woche in der letzten Zeit denken: An wievielen von den üblichen 6 Werktagen montags bis samstags hören Sie im allgemeinen Sendungen vom

Und wie ist es mit Sendungen vom

INT: Radio-Karten einzeln nacheinander auf Blatt 6 A vorlegen.

Anzahl der genannten Werktage (1 bis 6) im betreffenden Kästchen eintragen.

Falls ein Sender „so gut wie nie“ gehört wird, eine Null (=0) eintragen.

Nun folgt die Erfassung der Hörhäufigkeit für alle Sender im WHK.

Hier ein Beispiel:

FR3 Wenn Sie an eine normale Woche in der letzten Zeit denken:

An wie vielen von den üblichen Wochentagen Montags - Samstags

hören Sie im allgemeinen $\mathbf{w} \mathbf{d} \mathbf{r}$ zwei ?

INT: Anzahl Tage eintragen !!

Höre im allgemeinen nicht $<<$ null $>>$ eingeben

$0-6$

Die Antwortmöglichkeiten werden den Befragten beim Face-to-face-Interview auf einer Karte gezeigt (siehe Abb. 6 obere Hälfte). Der Interviewer trägt die Antworten in den in der Abb. 6 untere Hälfte eingefügten Fragebogenteil in das zu jedem Sender gehörige Feld 6 ein. Nach der Befragung werden die Fragebogendaten zur Hörhäufigkeit codiert. Beispielsweise findet sich unter der Feldnummer 159 die Variable „Hörhäufigkeit“ des Senders AlsterRadio mit Ausprägungen zwischen 0 und 8.

Vor 1987 wurde die Hörhäufigkeit detaillierter pro (Tages-) Zeitabschnitt pro Sender abgefragt, um die Hörerschaft pro (Tages-)Zeitabschnitt (HpTZ) zu ermitteln. Folgender Unterschied ist im Vergleich zwischen den Variablen Hörhäufigkeit pro Zeitabschnitt pro Sender (1975 bis 1986) und Hörhäufigkeit pro Sender (ab 1987) erkennbar: Vor 1987 wurden zu neun (Tages-) Zeitabschnitten alle Personen aus dem weitesten Hörerkreis (WHK) gefragt, an wie vielen Werktagen in einer normalen Woche bestimmte Einzelsender gehört wurden (Abb. 7).

Vom Interviewer wurden daraufhin die entsprechenden Antworten von $0=$ nie zu dieser Zeit bis $6=$ an allen Werktagen für jeden Zeitabschnitt in den Fragebogen eingetragen (Abb. 8, Feld 23). Beispielsweise wurde für die Zeit zwischen $7.00 \mathrm{Uhr}$ und 8.00 Uhr die Anzahl der Hörtage in einer normalen Woche ermittelt. ${ }^{16}$

Die Media-Analysen von 1975 (rechts) und 1985 (links) beinhalteten dasselbe Abfrageprinzip. Dabei unterschieden sich aber die abgefragten Zeitabschnitte.

Die Anzahl der erhobenen Zeitabschnitte pro Tag war nicht durchgängig konstant. Zwischen 1981 und 1986 wurden neun Zeitabschnitte abgefragt: Vor 7.00 Uhr, 7.00 bis

16 Zusätzlich codiert wurden jeweils $7=$ keine Angabe und $8=$ nicht WHK (Abb. 11). 
Abbildung 6: Erinnerungshilfe (eigene Darstellung), Fragebogen und Codierung (eigene Darstellung) der Frequenzabfrage (Hörbäufigkeit pro Woche): Fragebogen MA_99_EM (ma_99_EM_frb.pdf: 44) und Codeplan MA 99 EM (ma_99_EM_cdb.pdf: 76) (eigene Darstellung)

An den 6 Wochentagen Montag bis Samstag

Höre ich im Allgemeinen vom Sender Radio:

An 6 Tagen $=$ an jedem Tag

an 5 Tagen

an 4 Tagen

an 3 Tagen

an 2 Tagen

an 1 Tage

0 nie $=$ an keinem Tag

\begin{tabular}{|c|c|c|c|c|c|c|c|c|}
\hline \multicolumn{2}{|l|}{ Split } & \multirow{3}{*}{$\begin{array}{l}\text { B-Feld } \\
(+100)\end{array}$} & \multicolumn{6}{|c|}{ Antworten } \\
\hline 3 & $4^{2}$ & & \multicolumn{2}{|l|}{4} & \multicolumn{3}{|l|}{5} & \multirow{2}{*}{$\begin{array}{l}6 \\
\text { an } \\
6 \\
\text { Tagen }\end{array}$} \\
\hline$\downarrow$ & - Sender & & $\begin{array}{l}\text { noch nie } \\
\text { gehört }\end{array}$ & $\begin{array}{l}\text { schon } \\
\text { mal } \\
\text { gehört }\end{array}$ & $\begin{array}{l}\text { innerhalb } \\
\text { der } \\
\text { letzten } 2 \\
\text { Wochen }\end{array}$ & $\begin{array}{l}2-4 \\
\text { Wochen } \\
\text { her }\end{array}$ & $\begin{array}{l}\text { länger } \\
\text { her }\end{array}$ & \\
\hline 401 & \multicolumn{2}{|c|}{ ANTENNE BAYERN } & $\square 2$ & $\square 1$ & $\square 1$ & $\square 2$ & $\square 3$ & \\
\hline 402 & Bayerischer & Bayern 1 & $\square 2$ & $\square 1$ & $\square 1$ & $\square 2$ & $\square 3$ & \\
\hline 403 & Rundfunk & Bayern 2 Radio & $\square 2$ & $\square 1$ & $\square 1$ & $\square 2$ & $\square_{3}$ & \\
\hline 404 & & Bayern 3 & $\square 2$ & $\square 1$ & $\square 1$ & $\square 2$ & $\square 3$ & \\
\hline 405 & & Bayern 4 Klassik & $\square 2$ & $\square 1$ & $\square 1$ & $\square 2$ & $\square 3$ & \\
\hline 406 & & B 5 aktuell & $\square 2$ & $\square 1$ & $\square 1$ & $\square 2$ & $\square 3$ & \\
\hline
\end{tabular}

Hörhäufigkeit 0 von 6 Werktagen

11 von 6 Werktagen

22 von 6 Werktagen

33 von 6 Werktagen

44 von 6 Werktagen

55 von 6 Werktagen

66 von 6 Werktagen

7 keine Angabe

8 innerhalb der letzten 2 Wochen nicht gehört

Felder zu:

Zeitfilter Hörhäufigkeit

$\begin{array}{lll}91 & 156 & \text { delta radio } \\ 92 & 157 & \text { RADIO NORA } \\ 93 & 159 & \text { R.SH Radio Schleswig-Holstein } \\ 94 & 159 & \text { AlsterRadio }\end{array}$


Abbildung 7: Frequenzabfrage (Hörbäufigkeit pro Zeitabschnitt pro Woche): Fragebogen MA_1985 (ma_85_frb.pdf: 45) (eigene Darstellung)

24 INT: Blatt 24 aufschlagen!

Falls „innerhalb der letzten zwei Wochen“ zu einer Zeit gehört wurde:

Wenn Sie an eine normale Woche in der letzten Zeit denken: An wievielen von den 6 Werktagen montags bis samstags hören Sie im allgemeinen in der Zeit vor/zwischen .... Uhr und .... Uhr den ....?

Und wie ist es zu dieser Zeit mit dem ....?

INT: Mit der bei Frage 23 zuerst genannten Zeit beginnen und Radio-Karten einzeln nacheinander auf Blatt 24 A vorlegen. Alle bei Frage 23 genannten Zeiten durchgehen und für jede Zeit Radio-Karten einzeln nacheinander auf Blatt 24 A vorlegen!

Zahl der genannten Werktage (1 bis 6) im betreffenden Kästchen eintragen.

Falls ein Sender zu einer Zeit „nie“ gehört wird, eine Null (=0) eintragen.

Weiter mit Frage 25 auf der Rückseite des Kästchen-Schemas.

Tabelle 2: Konstruiertes Radio-Wochenmodell zur Erklärung des Methodeneffekts bei der Umkodierung der maximalen Zeitabschnittsdaten zur Hörbäufigkeit pro Woche

\begin{tabular}{cccccccccc}
\hline & Mo & Di & Mi & Do & Fr & Sa & $\begin{array}{c}\text { Maximale } \\
\text { Hörhäufigkeit } \\
\text { pro Zeitabschnitt } \\
\text { pro Woche }\end{array}$ & $\begin{array}{c}\text { Hör- } \\
\text { häufigkeit } \\
\text { pro } \\
\text { Woche }\end{array}$ \\
\hline Person 1 & $\begin{array}{c}7.00-8.00 \\
8.00-10.00\end{array}$ & 1 & 1 & 1 & 1 & 1 & 1 & 6 Tage & 6 Tage \\
Person 2 & $\begin{array}{c}7.00-8.00 \\
8.00-10.00\end{array}$ & 1 & 1 & 1 & 1 & 1 & & 5 Tage & 6 Tage \\
\hline
\end{tabular}

8.00 Uhr, [...], 18.00 bis 20.00 Uhr und nach 20.00 Uhr (Abbildung 9). Vorher waren es zum Teil nur 7 dieser 9 Abschnitte, die Fragen nach der Zeit vor 7.00 Uhr und nach 20.00 Uhr waren nicht immer dabei (z. B. 1977).

Seit 1987 spielen diese Tageszeiten keine Rolle mehr und es wird nur noch nach der generellen Radionutzung von WHK-Sendern gefragt.

Es gilt zu prüfen, ob die entsprechenden Daten miteinander verknüpft werden können: Es ist annäherungsweise möglich, die älteren Daten in das schmalere Korsett der aktuelleren Erhebungen zu zwängen. Dazu kann bei den Daten von 1975 bis 1986 der maximale Tagewert aus den Zeitabschnittswerten herangezogen werden. Es ist jedoch davon auszugehen, dass dieser eher zu klein sein wird. Dem liegt folgende Problematik zugrunde, wie anhand Tabelle 2 mit konstruierten Nutzerdaten demonstriert wird. Angenommen jemand hört in einer normalen Woche von Montag bis Samstag regelmäßig nach dem Aufstehen in der Zeit zwischen 7.00 bis 8.00 Uhr Radio, so würde das in der Wochenstatistik wie bei Person 1 dargestellt aussehen. Der maximale Wert pro Zeitab- 
Abbildung 8: links Frequenzabfrage (Hörbäufigkeit pro Zeitabschnitt pro Woche) Fragebogen MA_1985 (ma_85_frb.pdf:46)

rechts: Fragebogen MA_1975 (ma_75_frb.pdf: 24)

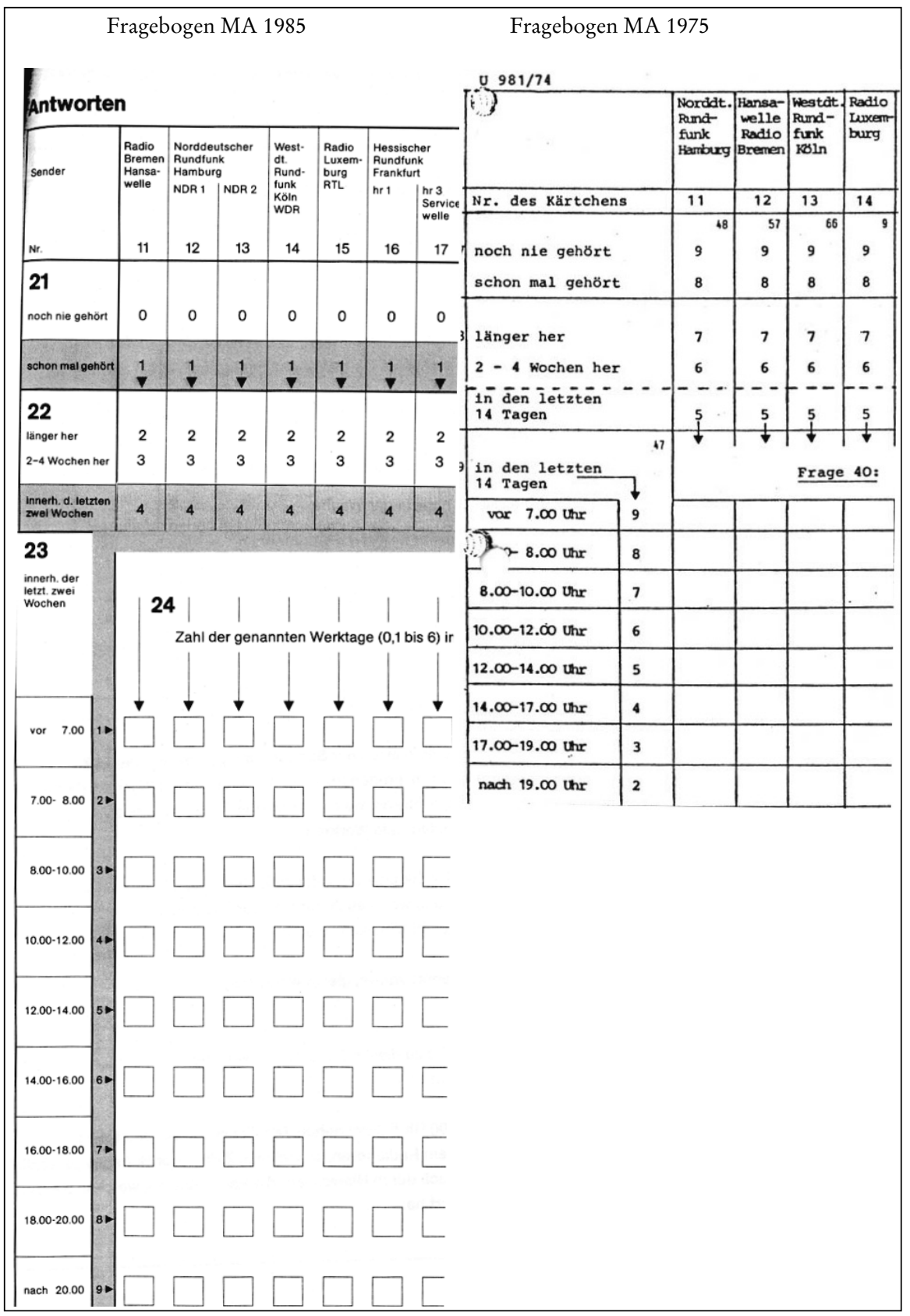


Abbildung 9: Codierung der Frequenzabfrage (Hörbäufigkeit pro Zeitabschnitt pro Woche): Codeplan MA_85 (ma_85_cdb.pdf: 100)

(eigene Darstellung)

\begin{tabular}{|c|c|c|c|}
\hline \multicolumn{4}{|c|}{ Hörhäufigkeit pro Zeitabschnitt pro Sender } \\
\hline \multicolumn{4}{|l|}{$\begin{array}{l}0 \text { nie zu dieser Zeit } \\
1 \text { an einem Werktag } \\
2 \text { an zwei Werktagen } \\
3 \text { an drei Werktagen } \\
4 \text { an vier Werktagen } \\
5 \text { an fünf Werktagen } \\
6 \text { an allen Werktagen } \\
7 \text { keine Angabe } \\
8 \text { nicht WHK }\end{array}$} \\
\hline & $\begin{array}{l}\text { Hansaw. } \\
\text { Radio } \\
\text { Bremen }\end{array}$ & $\begin{array}{l}\text { Nordd. } \\
\text { Rundf. } \\
\text { NDR1 }\end{array}$ & $\begin{array}{l}\text { Nordd. } \\
\text { Rundf. } \\
\text { NDR2 }\end{array}$ \\
\hline vor $7.00 \mathrm{Uhr}$ & 3929 & 3930 & 3931 \\
\hline $7.00-8.00 \mathrm{Uhr}$ & 3946 & 3947 & 3948 \\
\hline $8.00-10.00 \mathrm{Uhr}$ & 3963 & 3964 & 3965 \\
\hline $10.00-12.00 \mathrm{Uhr}$ & 3980 & 3981 & 3982 \\
\hline $12.00-14.00 \mathrm{Uhr}$ & 3997 & 3998 & 3999 \\
\hline $14.00-16.00 \mathrm{Uhr}$ & 4014 & 4015 & 4016 \\
\hline $16.00-18.00 \mathrm{Uhr}$ & 4031 & 4032 & 4033 \\
\hline $18.00-20.00 \mathrm{Uhr}$ & 4048 & 4049 & 4050 \\
\hline nach $20.00 \mathrm{Uhr}$ & 4065 & 4066 & 4067 \\
\hline
\end{tabular}

schnitt wäre 6 und würde der tatsächlichen Hörhäufigkeit pro Sender in einer normalen Woche entsprechen. Sollte jemand aber samstags ein wenig länger Schlafen (Person 2) und entsprechend später das Radio Einschalten ( 8.00 bis $10.00 \mathrm{Uhr}$ ) würde der maximale Zeitabschnittswert nur noch bei 5 liegen und somit kleiner sein als der reale Wert 6 Tage. Der eigentliche Wert 6 kann aber deshalb nicht ermittelt werden, weil - abweichend zu dem konstruierten Wochenmodell in Tabelle 2 - bei dem älteren Modell keine Angaben zu den einzelnen Wochentagen, sondern nur zu einer "normalen Woche“ vorliegen. Letztlich ist es aber möglich, die Daten vor und ab 1987 in eine Variable zu „zwängen“, der beschriebene Methodeneffekt sollte aber beachtet werden.

\subsection{Tagesablauf}

Stichtagsbezogene Nutzungsaktivitäten zum gestrigen Tag wurden prinzipiell schon 1975 erhoben. Seit 1987 erfolgt dies in einer detaillierteren Form (Buß 1998: $77 f f$.$) .$

Von allen Befragten wird seit 1987 bei der Abfrage des Tagesablaufs für den jeweils gestrigen Tag von 5 bis 24 Uhr ermittelt, welche Tätigkeit zu jeder Viertelstunde ausgeübt wurde (vgl. Akinci 2004). „Die Interviews werden nach Stichtagen gleichmäßig über die Woche verteilt, so dass in der Gesamtstichprobe die einzelnen Wochentage in etwa gleichem Umfang repräsentiert sind“ (Koschnick 2004b). 
Abbildung 10: Tagesablauf-Abfrage (Akinci 2004: 2)

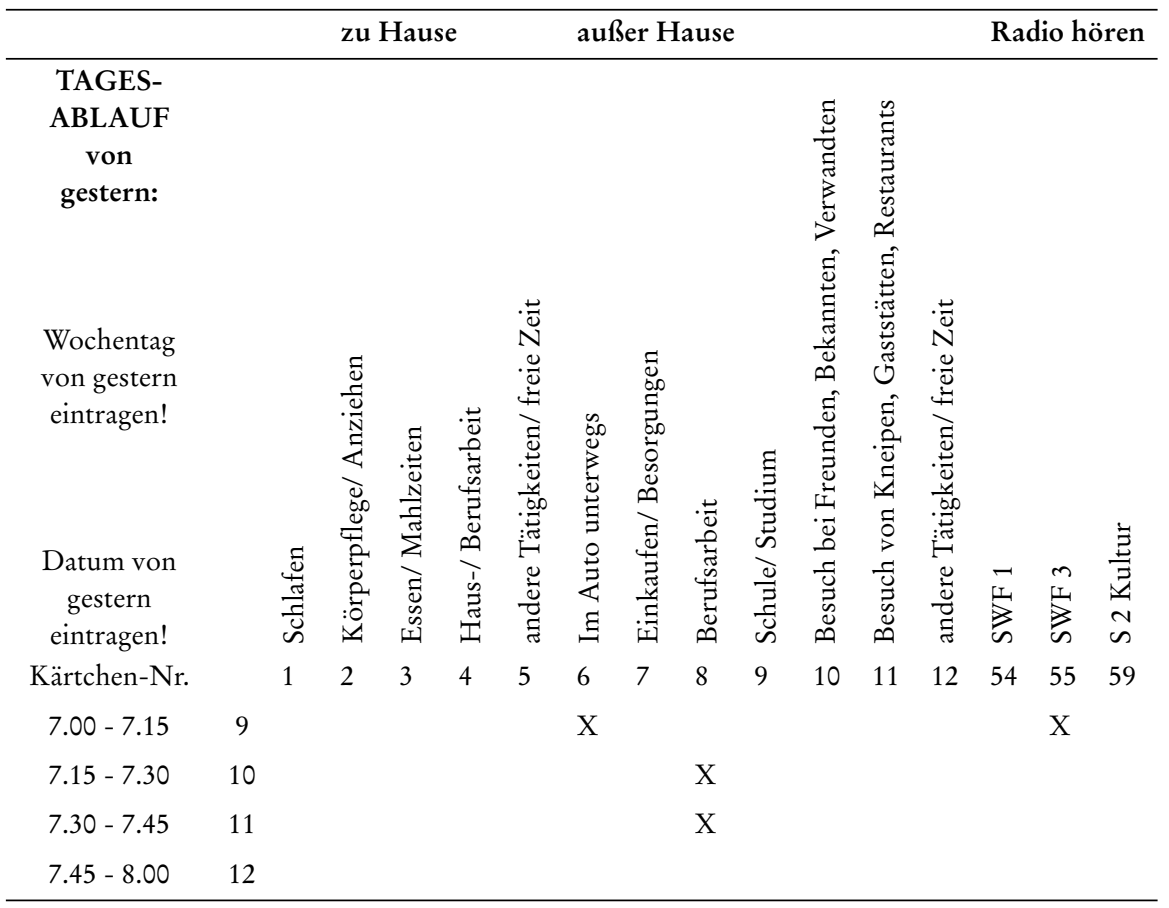

Wie in Abbildung 10 dargestellt, gehören zu den Aktivitäten unterschiedliche Leittätigkeiten „im Haus" sowie „außer Haus" und beinhalten Kategorien wie bspw. Schlafen, Beruf, Hausarbeit und Einkaufen. Kern dieser Abfrage ist jedoch die Ermittlung der Radionutzung jedes Einzelsenders. Diese detaillierte Erhebungsform, in der für jede Viertelstunde Nutzungsaktivitäten verbunden mit Leittätigkeiten erhoben wurden, wird seit 1987 kontinuierlich durchgeführt. Vorher waren die abgefragten Zeitabschnitte größer (halbe oder volle Stunden) und Leitaktivitäten wurden nicht abgefragt (ausführlicher im nächsten Abschnitt).

Der Interviewer unterstützt den Befragten in einem Gespräch, sich mit Hilfe der eben genannten Leittätigkeiten an den Verlauf des gestrigen Tages zu erinnern. Auf diese Weise kann nicht nur das Hören unterschiedlicher Sender eines Intervalls erfasst werden, sondern auch, welche Sender während welcher anderen Tätigkeit gehört wurde (Akinci 2004: 2). Es werden also auch parallel stattfindende Aktivitäten erfasst.

Der Abbildung 11 lassen sich die Intervieweranweisungen und die vorzulesenden Abschnitte für die persönlich-mündlich durchgeführten Befragungen entnehmen. Der Interviewer muss für jede Viertelstunde des davorliegenden Tages alle Tätigkeiten in den in Abb. 10 ausschnittsweise gezeigten Fragebogen eintragen.

Beim telefonischen Interview sehen die Instruktionen zur Tagesablauf-Befragung wie in Abb. 12 dargestellt aus. Für jede Viertelstunde von 5.00 - 24.00 öffnet sich bei der Befragung ein Eingabefenster in das mindestens eine Leittätigkeit bzw. die Nutzung eines Mediums eingetragen werden muss.

Codiert werden die Tagesablaufvariablen als sog. „Dummy“-Variablen, d. h. für jede 
Abbildung 11: Fragebogenhandling der Tagesablauf-Abfrage beim persönlichmündlichen Interview: Fragebogen MA_1999_EM (ma_99_EM_frb. pdf: 9) (eigene Darstellung)

11 INT: Die laut Frage 5 bereitgehaltenen „innerhalb der letzten 2 Wochen gehört“ RadioKarten vor dem Befragten ausbreiten. Liste Y ebenfalls vor dem Befragten aufgeschlagen liegen lassen.

Im Rahmen dieser Untersuchung möchten wir gerne einmal feststellen, was die Bevölkerung zu bestimmten Tageszeiten macht. Hierfür möchte ich gerne den gestrigen Tag einmal mit Ihnen durchgehen und zwar beginnend mit 5.00 Uhr morgens.

Bis wann Sie geschlafen haben, was Sie im Laufe des gestrigen Tages bis $24.00 \mathrm{Uhr}$ nachts alles gemacht haben. Dabei möchte ich natürlich auch gerne erfahren, ob und wann Sie gestern Radio oder Schallplatten, Tonband, Kassetten, CDs gehört und ob und wann Sie gestern fern- oder Video gesehen oder sich mit dem PC beschäftigt haben.

Beim Radiohören interessieren wir uns genau für die einzelnen Sender und Programme. Denken Sie bitte auch an Zeiten, in denen Sie nur kurz oder nebenbei Radio gehört haben. Ich möchte nur wissen, wie das gestern war, also auch dann, wenn der gestrige Tag nicht besonders typisch war.

\section{ACHTUNG INTERVIEWER:}

- Die ausgebreiteten Radio-Karten und die Liste Y mit den Rundtunksendern und Radioprogrammen sollen für den Befragten eine Erinnerungshilfe sein.

- Die Tätigkeit „Autofahren“ gilt auch für Beifahrer.

- Unbedingt bei jeder Tätigkeit (auch Außer-Haus) nachfragen, ob dabei Radio gehört wurde.

- Die Tätigkeit „schlafen“ schließt auch „im Bett liegen“, „sich ausruhen“ ein, dabei kann auch Radio gehört werden.

Bei den Tätigkeiten zu Hause und außer Haus:

- Für jede Viertelstunde muß eine Angabe gemacht werden.

- Mehrfachnennungen sind möglich.

- Nicht zuordenbare Tätigkeiten sind unter „andere Tätigkeiten /Freie Zeit“ einzutragen.

- Bei Radioprogrammen sind Doppelnennungen innerhalb der gleichen Viertelstunde möglich. Es wird z.B. innerhalb der gleichen Viertelstunde von einem Radioprogramm auf das andere umgeschaltet.

- Es kann gleichzeitig Radio gehört und ferngesehen werden.

INT: Jetzt im nachfolgenden Tagesablauf mit 5.00 Uhr morgens beginnend bis $\mathbf{2 4 . 0 0}$ Uhr nachts viertelstundenweise in den entsprechenden Kästchen genau markieren, was der Befragte gestern von wann bis wann alles gemacht hat. Sind bei einer Tätigkeit (z.B. Berufsarbeit außer Haus) viele 1/4 Std.-Kästchen hintereinander zu markieren, kann ein Lineal hilfreich sein. Bitte beachten Sie zum Ausfüllen des Tagesablaufs auch die besonderen Hinweise im Interviewer-Anschreiben. 
Abbildung 12: Fragebogenhandling und Dateneingabe bei der Tagesablauf-Abfrage beim telefonischen Interview: Fragebogen MA_2000_EM II (ma_00_ EM_II_frb.pdf: $7 f$.)

Im Rahmen dieser Untersuchung soll unter anderem herausgefunden werden, zu welchen Zeiten welche Radiosender gehört werden und wann ferngesehen wird. Daran kann man sich erfahrungsgemäss am besten erinnern, wenn man sich überlegt, was man den Tag über im einzelnen gemacht hat:

wann man aufgestanden ist, wann man zur Arbeit oder zum Einkaufen gegangen ist usw.

Wie war das gestern bei Ihnen?

INT: ACHTUNG: Der Tagesablauf kann nicht unterbrochen und nur einmal im Interview aufgerufen werden.

Tagesablaufschema - Bildschirmmatrix:

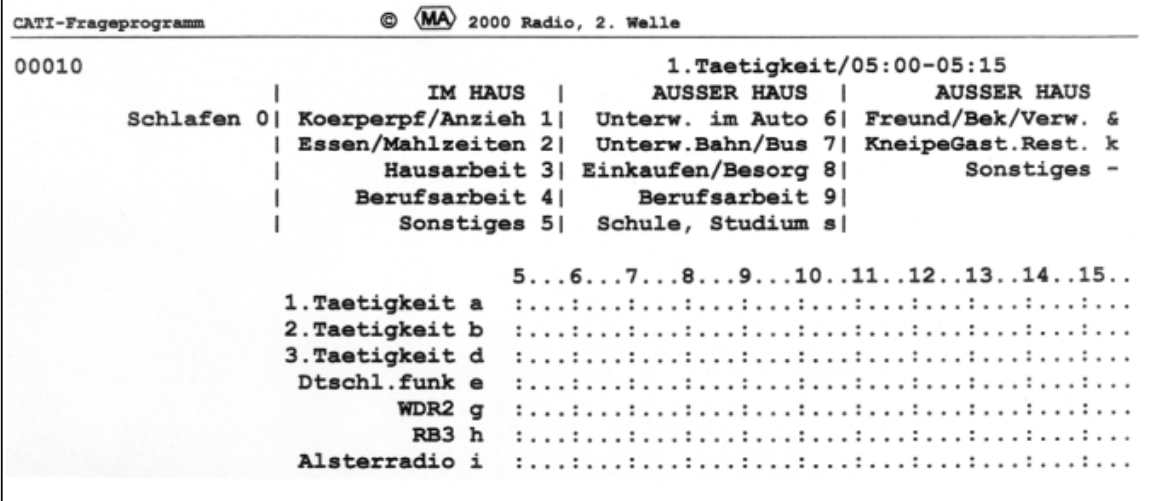

Abbildung 13: Tagesablauf-Abfrage: Fragebogen MA_1975 (ma_75_frb.pdf: 23) (eigene Darstellung)

41.a) Und wie war das gestern (Montaginterview: vorgestern) nun im einzelnen: Wann haben Sie zum ersten Mal Radio gehört?

INTERVIEWER: Für jede Tageszeit, zu der gehört wurde, sofort nachfragen: Liste 27 vorlegen!

41.b) Und welchen Sender, welches Programm hatten Sie eingeschaltet?

Hier sind Kärtchen mit dem Namen der Rundfunksender und Programme, an denen Sie die Sender erkennen können:

INTERVIEWER: In freiem Gespräch alle Tageszeiten durchgehen und möglichst genau feststellen und kringeln, zu welchen Tageszeiten der Befragte gestern (Montaginterview: vorgestern) Radio gehört hat und welchen Sender, welches Programm der Befragte gestern (Montaginterview: vorgestern) zu den entsprechenden Zeiten eingeschaltet hatte. 
Abbildung 14: Tagesablaufraster: Fragebogen MA_1975 (ma_75_frb.pdf: 24)

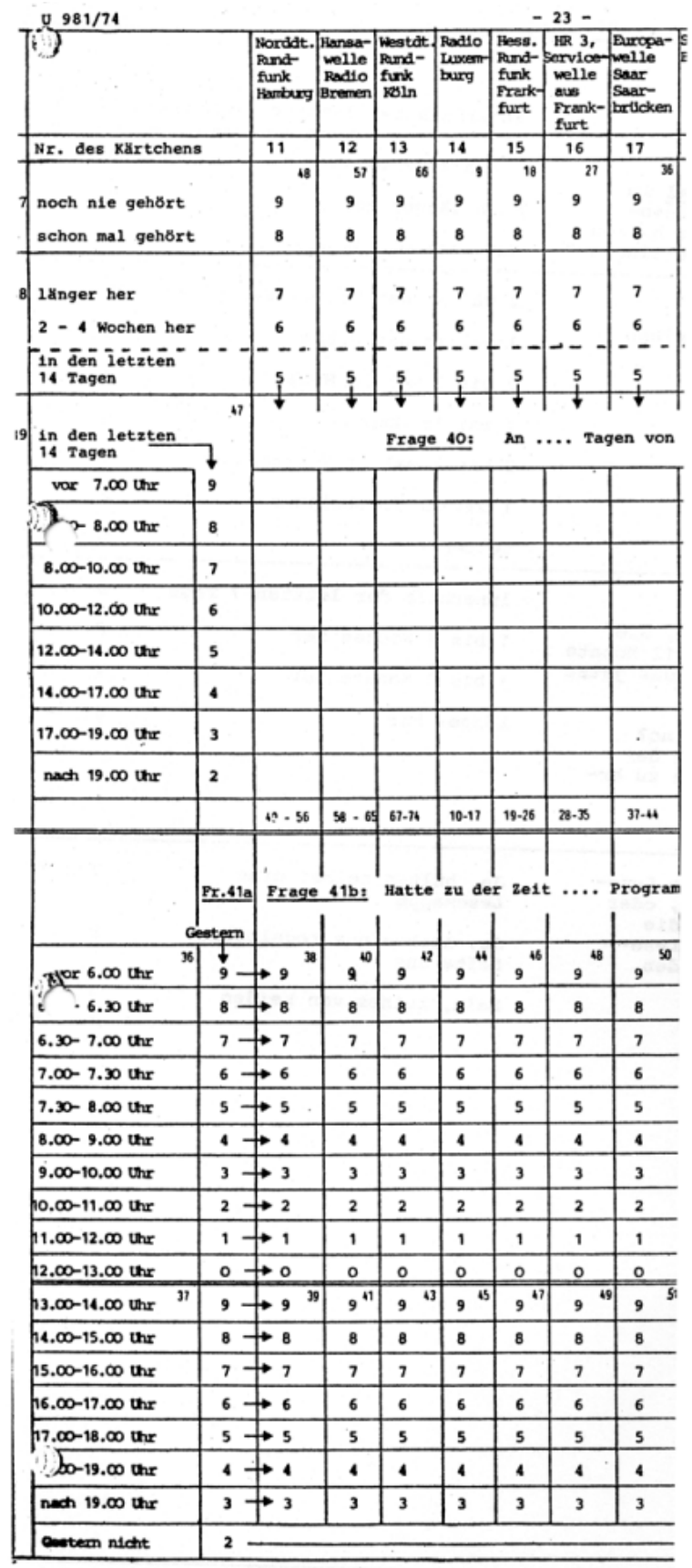


Viertelstunde und jede Tätigkeit gibt es eine Variable. Auf diese Weise ist jeweils abzulesen, ob die Tätigkeit in dieser Viertelstunde ausgeführt wurde $(1=\mathrm{ja})$ oder nicht $(0=$ nein).

Auch beim älteren MA-Erbebungsmodell von 1975 bis 1986 gab es Fragen zum jeweils gestrigen Tagesablauf (am Montag zu vorgestern, zum Sonntag wurde nicht befragt). Dieses „freie Gespräch“ beschränkte sich jedoch auf das Einholen von Nutzungswerten zu den elektronischen Medien Radio und Fernsehen (Abb. 13), Leittätigkeiten im und außer Haus wurden (noch) nicht abgefragt.

Wie in Abbildung $14 \mathrm{zu}$ sehen, wurde die Radiorezeption pro Zeitabschnitt abgefragt. Für die Zeit zwischen 6.00 und $8.00 \mathrm{Uhr}$ waren das Halbstunden, danach bis 19.00 Uhr volle Stunden. Ab 1979 wurde der Tagesablaufbereich vergrößert, so dass die halben ab 5.00 Uhr und die vollen Stunden bis 22.00 Uhr thematisiert wurden.

Da die im Fragebogen 1975 beschriebenen Tagesablaufdaten nicht im dazugehörigen Codeplan ausgewiesen sind, wird im Folgenden auf diejenigen des Jahres 1976 (Abbildung 1) verwiesen. Die in der Abbildung 15 als Merkmal C bezeichneten Daten, wurden auch als „gestern pro Zeitabschnitt pro Sender bezeichnet" und codiert mit $1=$ gestern ja, $2=$ gestern nein und $3=$ nicht WHK.

Es ist möglich, die Viertelstundendaten des aktuellen Erhebungsmodells in die (Halb-)

Abbildung 15: Codeplan MA 1976 (ma_76_cdb.pdf: 79, 78)

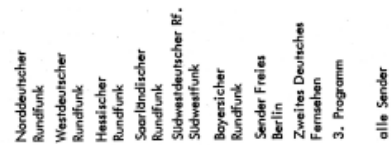

$\begin{array}{lllllllllll}\text { Halbbyte-Nr. } & & & & & & & & & \\ \text { Merkmal A } & 599 & 611 & 633 & 655 & 677 & 699 & 721 & 743 & 765 & \\ \text { Merkmal B } & & & & & & & & & & \\ \text { vor } 17.00 \text { Uhr } & 590 & 612 & 634 & 656 & 678 & 700 & 722 & 744 & 766 & \\ 17.00 \text { bis } 17.30 \text { Uhr } & 591 & 613 & 635 & 657 & 679 & 701 & 723 & 745 & 767 & \\ 17.30 \text { bis } 18.00 \text { Uhr } & 592 & 614 & 636 & 658 & 680 & 702 & 724 & 746 & 768 & \\ 18.00 \text { bis } 18.30 \text { Uhr } & 593 & 615 & 637 & 659 & 681 & 703 & 725 & 747 & 769 & \\ 18.30 \text { bis } 19.00 \text { Uhr } & 594 & 616 & 638 & 660 & 682 & 704 & 726 & 748 & 770 & \\ 19.00 \text { bis } 19.30 \text { Uhr } & 595 & 617 & 639 & 661 & 683 & 705 & 727 & 749 & 771 & \\ 19.30 \text { bis } 20.00 \text { Uhr } & 596 & 618 & 640 & 662 & 684 & 706 & 728 & 750 & 772 & \\ & & & & & & & & & & \\ \text { Merkmal C } & & & & & & & & & & \\ \text { vor } 17.00 \text { Uhr } & 597 & 619 & 641 & 663 & 685 & 707 & 729 & 751 & 773 & 787 \\ 17.00 \text { bis } 17.15 \text { Uhr } & 598 & 620 & 642 & 664 & 686 & 708 & 730 & 752 & 774 & 788 \\ 17.15 \text { bis } 17.30 \text { Uhr } & 599 & 621 & 643 & 665 & 687 & 709 & 731 & 753 & 775 & 789 \\ 17.30 \text { bis } 17.45 \text { Uhr } & 600 & 622 & 644 & 666 & 668 & 710 & 732 & 754 & 776 & 790 \\ 17.45 \text { bis } 18.00 \text { Uhr } & 601 & 623 & 645 & 667 & 669 & 711 & 733 & 755 & 777 & 791 \\ 18.00 \text { bis } 18.15 \text { Uhr } & 602 & 624 & 646 & 668 & 690 & 712 & 734 & 756 & 778 & 792 \\ 18.15 \text { bis } 18.30 \text { Uhr } & 603 & 625 & 647 & 669 & 691 & 713 & 735 & 757 & 779 & 793 \\ 18.30 \text { bis } 18.45 \text { Uhr } & 604 & 626 & 648 & 670 & 692 & 714 & 736 & 758 & 780 & 794 \\ 18.45 \text { bis } 19.00 \text { Uhr } & 605 & 627 & 649 & 671 & 693 & 715 & 737 & 759 & 781 & 795 \\ 19.00 \text { bis } 19.15 \text { Uhr } & 606 & 628 & 650 & 672 & 694 & 716 & 738 & 760 & 782 & 796 \\ 19.15 \text { bis } 19.30 \text { Uhr } & 607 & 629 & 651 & 673 & 695 & 717 & 739 & 761 & 783 & 797 \\ 19.30 \text { bis } 19.45 \text { Uhr } & 608 & 630 & 652 & 674 & 696 & 718 & 740 & 762 & 784 & 798 \\ 19.45 \text { bis } 20.00 \text { Uhr } & 609 & 631 & 653 & 675 & 697 & 719 & 741 & 763 & 785 & 799 \\ \text { Merkmal D } & 610 & 632 & 654 & 676 & 698 & 720 & 742 & 764 & 786 & 600\end{array}$

C. Gestern pro Zeitabschnitt und Sender

1 gestern ja (3 Ausprägungen) 
Stundendaten der älteren Erhebungen umzukodieren. Dies beinhaltet jedoch einen Informationsverlust für die neueren Daten. Wichtiger ist es aber, einen denkbaren Methodeneffekt bei der Dateninterpretation zu berücksichtigen. Für ein paar Sender ist somit der Tagesablauf innerhalb des Zeitfensters von 6.00 Uhr bis $19.00 \mathrm{Uhr}$ in (Halb-) Stunden von 1976 an analysierbar.

\subsection{Zusammenfassung: Sender-Abfragemodelle}

In Tabelle 3 werden die Nutzungsmöglichkeiten der vier Kernkomplexe der beiden eingesetzten Erhebungsmodelle (1975 bis 1986 bzw. ab 1987 bis heute) für Longitudinalstudien zusammenfassend miteinander verglichen. Problemlos lassen sich die Daten zu General- und Zeitfilter von 1975 bis heute verknüpfen. Lange Reanalysen zur Frequenzabfrage lassen sich nur sehr eingeschränkt bewerkstelligen, Tagesablaufstudien sind nach Umkodierungen der aktuelleren Viertelstundendaten auf (Halb-) Stunden für einen begrenzten Tageszeitraum (6.00 - 19.00 Uhr) unter Berücksichtigung eines Methodeneffekts eingeschränkt möglich.

Tabelle 3: Nutzungsmöglichkeiten senderspezifischer Radiodaten von 1975 bis heute: Vergleich zwischen altem und aktuellem Erhebungsmodell

\begin{tabular}{|c|c|c|c|c|}
\hline $\begin{array}{l}\text { Abfrage- } \\
\text { block }\end{array}$ & $\begin{array}{l}\text { Aktuelles Ab- } \\
\text { fragemodell } \\
\text { (seit 1987) }\end{array}$ & $\begin{array}{l}\text { Altes Abfrage- } \\
\text { modell } \\
(1975 \text { bis 1986) }\end{array}$ & $\begin{array}{l}\text { Methodischer } \\
\text { Vergleich }\end{array}$ & $\begin{array}{l}\text { Längsschnittliche } \\
\text { Nutzbarkeit }\end{array}$ \\
\hline $\begin{array}{l}\text { General- } \\
\text { filter }\end{array}$ & $\begin{array}{l}\text { Schon mal ge- } \\
\text { hört? }\end{array}$ & $\begin{array}{l}\text { Schon mal ge- } \\
\text { hört? }\end{array}$ & identisch & $\begin{array}{l}\text { Problemlos } \\
1975 \text { bis heute }\end{array}$ \\
\hline $\begin{array}{l}\text { Für alle } \\
\text { schon mal } \\
\text { gehörten } \\
\text { Sender: } \\
\text { Zeitfilter }\end{array}$ & $\begin{array}{l}\text { „wann zuletzt?“ } \\
\text { länger her/ } \\
\text { 2-4 Wochen her/ } \\
\text { letzte } 14 \text { Tage }\end{array}$ & $\begin{array}{l}\text { „wann zuletzt?“ } \\
\text { länger her/ } \\
\text { 2-4 Wochen her/ } \\
\text { letzte } 14 \text { Tage }\end{array}$ & identisch & $\begin{array}{l}\text { Problemlos } \\
1975 \text { bis heute }\end{array}$ \\
\hline $\begin{array}{l}\text { Letzte } 14 \\
\text { Tage ge- } \\
\text { hört: } \\
\text { Frequenz }\end{array}$ & $\begin{array}{l}\text { Hörhäufigkeit: } \\
\text { „wie viele der } \\
\text { sechs Werkta- } \\
\text { ge?“ }\end{array}$ & $\begin{array}{l}\text { Hörhäufigkeit } \\
\text { pro Zeitab- } \\
\text { schnitt: „wie } \\
\text { viele der sechs } \\
\text { Werktage?“ }\end{array}$ & $\begin{array}{l}\text { Detaillierter } \\
\text { beim alten } \\
\text { Modell pro Zeit- } \\
\text { abschnitt (z. B. } \\
7.00-8.00 \text { Uhr } \\
\text { an } 0 \text { bis 6) }\end{array}$ & $\begin{array}{l}\text { Sehr Eingeschränkt } \\
\text { möglich } \\
1975 \text { bis heute: Besser } \\
1975 \text { bis } 1986+1987 \\
\text { - heute } \\
\text { (Deutlicher Metho- } \\
\text { deneffekt) }\end{array}$ \\
\hline $\begin{array}{l}\text { Alle Be- } \\
\text { fragten: } \\
\text { Tagesab- } \\
\text { lauf }\end{array}$ & $\begin{array}{l}\text { Hörer gestern } \\
\text { pro Viertelstun- } \\
\text { de (ja/ nein); an } \\
\text { Leittätigkeiten } \\
\text { gekoppelt }\end{array}$ & $\begin{array}{l}\text { Hörer gestern } \\
\text { pro Zeitab- } \\
\text { schnitt: (Halbe-) } \\
\text { Stunde (ja/ } \\
\text { nein); nicht an } \\
\text { Leittätigkeiten } \\
\text { gekoppelt }\end{array}$ & $\begin{array}{l}\text { Unterschiedliche } \\
\text { Zeiteinheiten }\end{array}$ & $\begin{array}{l}\text { Eingeschränkt mög- } \\
\text { lich: } 1976 \text { bis heute: } \\
\text { Besser } 1975 \text { bis } 1986+ \\
1987 \text { - heute } \\
\text { Viertelstundendaten } \\
\text { umkodieren in (Halb-) } \\
\text { Stundendaten (Infor- } \\
\text { mationsverlust, Me- } \\
\text { thodeneffekt). }\end{array}$ \\
\hline
\end{tabular}




\section{Abgeleitete Daten: Aggregationen und Segmentationen}

Neben den ursprünglichen Fragebogeninformationen lassen sich in den ausgelieferten Datensätzen der Media-Analyse auch abgeleitete Variablen finden. Dazu gehören Aggregationen, Nutzungswabrscheinlichkeiten, Kontaktsummen und Varianzen. Informationen zu diesen Daten lassen sich in den Codeplänen finden, in den Fragebögen nicht. Als klassisch abgeleitet können die vielen Aggregationen (Zusammenfassungen) bezeichnet werden. Sie bestehen i.d.R. aus Summen von Einzelwerten.

Nutzungswabrscheinlichkeiten werden mit einem Segmentationsprogramm berechnet (siehe Abschnitt 3.1) und sollen nach Mai (2003: 18) angeben können, wie viele Personen zukünftig an einem durchschnittlichen Tag (Hörer pro Tag), in einer bestimmten Stunde (Hörer pro Stunde) oder in einer durchschnittlichen Stunde Radio hören. Kontaktsummen und Varianzen werden auf Basis der Nutzungswahrscheinlichkeiten berechnet (Abschnitt 3.2).

Beim Großteil der zur Verfügung stehenden Variablen handelt es sich um in den Codeplänen als abgeleitet bezeichnete Daten. Eigentlich sollte man sie eher als aufbereitete bezeichnen, da die Auswahl der dafür verwendeten Methoden und Begriffe programmatisch geprägt geschieht. Aber aufgrund einer ansonsten eher verwirrenden Darstellung werden sie weiterhin abgeleitet genannt. Um deren aktuellen Anteil zu veranschaulichen, werden die Datenblöcke der MA 2000 Radio kurz beschrieben: Von den neun Teildateien beinhalten drei originäre Befragungsdaten. Teil A enthält unter anderem die Angaben zur Demografie (plus Besitz im Haushalt etc.), Teil A Eins die Tätigkeiten aus dem Tagesablauf und Teil $\mathrm{H}$ die Originärinformationen zur Mediennutzung. In den Teilen B bis $\mathrm{G}$ findet man nach bestimmten Konventionen aufbereitete Daten zum Werbeträgerkontakt $(\text { WTK })^{17}$ und zum Werbemittelkontakt $(\text { WMK })^{18}$. Hierbei

17 Ein Werbeträgerkontakt (WTK) ist jeder - auch flüchtige Kontakt - zwischen einer Person und einem Werbeträger (z. B. einer Zeitung/Zeitschrift, einem Fernseh- oder Hörfunksender). Der WTK im Hörfunk „gibt die Anzahl der Personen an, die von einem oder mehreren Werbeträgern mindestens einmal in irgendeiner Viertelstunde innerhalb einer Stunde einmal erreicht werden. In die Berechnung dieser Netto-Reichweite geht jede Person nur einmal ein, d. h. Doppelund Mehrfachkontakte werden nicht innerhalb einer Stunde berücksichtigt" (www.topradio. de, 2004). Eine Werbeträgerkontakt-Chance liegt vor, wenn mindestens eine Viertelstunde in einer werbungführenden Stunde gehört wird (Mai 2003). Da dieser Kontakt theoretisch auch bei nur „einer Viertelstunde nach einem Werbeblock erfolgen kann, spricht man hier von der Kontaktchance zum Werbeträger - Radio/ Sender" (www.rms.de, 2004a).

18 Ein Werbemittel-Kontakt (WMK) ist jeder - auch flüchtige Kontakt - zwischen einer Person und einem Werbemittel (z. B. einer Anzeige, einem Hörfunk- oder Werbefernsehspot). Der WMK im Hörfunk ,gibt die Anzahl der Personen an, die in einer durchschnittlichen Viertelstunde innerhalb einer Stunde erreicht werden. Die Hörer der durchschnittlichen Viertelstunde errechnen sich dabei aus dem arithmetischen Mittel der Addition der Hörer, die in den vier Viertelstunden einer Stunde mindestens einmal erreicht wurden“" (www.topradio.de, 2004). Der Werbemittelkontakt ist nach Koschnick (2004a) „die Größe, die angibt, wie viele Nutzer eines Mediums mit einem konkreten Werbemittel Kontakt hatten, d. h. mindestens irgendetwas darin wahrgenommen haben. Die Werbemittelkontaktchance (WMKC) verallgemeinert den Werbemittelkontakt, indem sie die Chance eines durchschnittlichen Werbemittels, d. h. aller Werbemittel in einem Medium, misst und dies als Wahrscheinlichkeit ausdrückt.“ Berechnet wird sie, ,indem man den WMK durch den WTK dividiert und mit 100 multipliziert. Das Ergebnis zeigt das prozentuale Verhältnis des WMK zum WTK. Eine Person, die vier Viertelstunden nutzt, hat somit eine Nutzungswahrscheinlichkeit von $100 \%$, eine Person, die nur eine Viertelstunde nutzt, hat eine Nutzungswahrscheinlichkeit von $25 \%$ “ (www.rms.de, 2004b). 
handelt es sich um für die Werbe- und Mediaindustrie abgeleitete Daten, die vor allem in Wabrscheinlichkeitswerte ( $p$-Werte) transformiert wurden und als Prognosemittel für die zukünftige Mediennutzung dienen und bei der Mediaplanung helfen sollen. Auf diese Weise wird es ermöglicht, in speziellen Planungsprogrammen Reichweiten bei mehreren Schaltungen auszuweisen.

\subsection{Ermittlung der Nutzungswahrscheinlichkeiten ( $p$-Werte) durch Segmentation}

Die Berechnung der Nutzungswahrscheinlichkeiten mittels Segmentation erfolgt jeweils für diejenigen Befragten, die zum weitesten Hörerkreis eines Senders gehören und wird in Abbildung 16 erklärt. Grob verkürzt kann man sagen, dass versucht wird, mit Hilfe statistischer Verfahren Nutzungsabstufungen zwischen 0 und 100 Prozent für die Hörer jedes Senders zu finden. Automatisch eine 0 bekommen dabei Nichthörer, die nicht zum weitesten Hörerkreis gehören, und 100 Prozent zuverlässige Stammhörer. Dazwischen werden mit Hilfe eines Segmentationsprogramms Personen mit ähnlichen Radionutzungsgewohnheiten und soziodemographischen Merkmalen auf Basis einer Diskriminanzanalyse gruppiert.

Den komplizierten Verarbeitungsprozess beschrieb Ernst 1978 mit folgenden Worten:

„Zwischen der Erhebung der Daten und dem Ausweis von Nutzungswahrscheinlichkeiten wird ein beachtlicher und notwendiger Aufwand an ,Verarbeitung' getrieben: es wird transformiert, egalisiert, redressiert, segmentiert und so weiter und den bereits zitierten interessierten ,Laien' überfällt gelegentlich ein diffuses Unbehagen, wenn er betrachtet und zu verstehen versucht, was da alles aufgewend $(\mathrm{t})$ et wird, um zu einer so einfachen Information zu kommen, die als , $\mathrm{p}$-Wert' bekannt ist. Wobei ich nicht unbedingt sicher bin, ob denn alle MA-Mitglieder imstande wären, einem weniger interessierten Laien zu erklären, was denn dieses ominöse ,p’ überhaupt bedeutet.“ (Ernst 1995: 90)

Mindestens drei Segmentationsprogramme sind im Laufe der Jahre in der MA eingesetzt worden ${ }^{19}$ : „Zunächst war es ein Programm, das Infratest, München, aus Frankreich übernommen hatte, dann ein ebenfalls aus Frankreich entlehntes Programm von ISBA und schließlich ein Programm, das IBM, Frankfurt/Main, für das Bureau Wendt, Hamburg, unter Verwendung der deutsch-französischen Erfahrungen geschrieben hat“ (Koschnick 2004c).

Der Werbemittelkontakt bei den Funkmedien in seiner reinsten Form ist gleich dem Kontakt mit dem konkreten Werbespot (Koschnick 2004a). Diese Information sei aus einem Metersystem wie dem der AGF/GfK-Fernsehforschung relativ leicht, aus Befragungen jedoch nur schwer zu gewinnen. Der durchschnittlichen Werbemittelkontaktchance entspricht die Blockreichweite in einer halben Stunde bzw. Stunde mit Werbung. Auch sie sei aus einem Metersystem recht leicht, aus Befragungen jedoch nur schwer zu gewinnen und entsprechend für das Fernsehen leichter herstellbar als für den Hörfunk.

19 Es muss noch recherchiert werden, wann die Segmentationsprogramme gewechselt wurden. Leider gibt es laut Auskunft der ARD Werbung \& Sales Services GmbH keine Publikation, aus der der Ablauf der Geschichte der Segmentation innerhalb der MA abgelesen werden kann. Diesbezügliche Anfragen bei der AG.MA blieben erfolglos. Auch Herr Koschnick konnte bei einem persönlichen Gespräch keine weiteren Details berichten. 
Abbildung 16: Die Berechnung der Nutzungswabrscheinlichkeiten mittels

Segmentation (Eigene Darstellung in Anlehnung an Mai 2003: 19)

\begin{abstract}
Die Berechnung der Nutzungswahrscheinlichkeiten
Die Berechnung der $\mathrm{p}$-Werte eines Senders erfolgt pro Werbestunde und getrennt für Männer und Frauen mit Hilfe der Segmentation.

Hierfür wird der weiteste Hörerkreis in Segmente unterteilt, die in sich möglichst homogen sind, z. B. niedrige und hohe Radionutzung. Die Segmente unterscheiden sich jedoch stark in ihrer Reichweite der jeweiligen Stunde. Die beiden so entstandenen Segmente werden nun ihrerseits in jeweils zwei Segmente unterteilt, die sich stark in ihrer Reichweite unterscheiden (z. B. jüngere und ältere Befragte). Wenn ein Segment nicht weiter teilbar ist, wird den Befragten dieses Segments als p-Wert die Reichweite in Prozent zugewiesen. Dieser Wert liegt zwischen 0 und $100 \%$ und gibt an, mit wie viel Prozent Wahrscheinlichkeit der Befragte in dieser Stunde über diesen Sender erreicht werden kann. Alle Befragten, die nicht zum weitesten Hörerkreis gehören, erhalten den p-Wert Null.

Die Auswahl der Segmentationsvariablen erfolgt durch ein Segmentationsprogramm, das aus gegebenen Variablen diejenigen aussucht, die am besten trennen.

Beispiel: Sender XY in der Uhrzeit 8.00 - 9.00 Uhr, Männer

Berufstätige Männer mit einer geringen Radionutzung aus dem WHK des Senders XY haben in der Zeit zwischen 8.00-9.00 Uhr eine Wahrscheinlichkeit von $18 \%$. Bei der Berechnung des Hörers pro Stunde gehen sie daher nicht mit ihrer Anzahl 90, sondern nur mit 18 \% davon (16) ein. Die Summe der Reichweiten der einzelnen Segmente ergibt wieder die Reichweite pro Stunde $(21+14+16+9=60)$.
\end{abstract}

\title{
Beispiel einer Segmentation
}

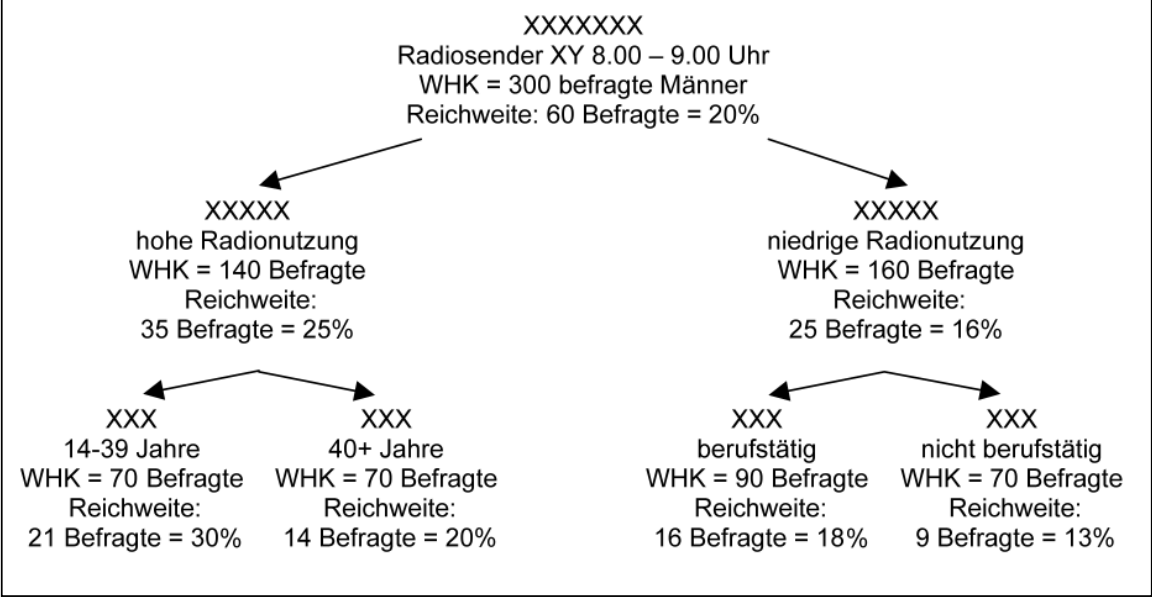

Die seit 1971 durchgeführten Segmentationsberechnungen beziehen sich auf das Erstellen von p-Werten für den Werbeträgerkontakt, WMK-Daten sind erst seit 1992 in den Media-Analysen enthalten. Da der p-Wert über die Jahre hinweg immer zwischen 0 und 100 Prozent (bis 1975: 0 bis $99 \%$ ) variierte, scheint auf den ersten Blick eine Vergleichbarkeit gegeben zu sein. Es muss jedoch beachtet werden, dass das Zustandekommen auf jeweils unterschiedlichen Verfahren basiert, die zum Teil sogar jährlich „optimiert“" wurden. 
Es ließe sich problemlos ein Datensatz mit Nutzungswahrscheinlichkeiten von 1972 bis heute erstellen, aber Ergebnisse möglicher Longitudinalstudien müssten an den Bruchstellen (Methodenwechsel: unterschiedliche Segmentationsverfahren) entsprechend vorsichtig interpretiert werden. Es bedarf letztlich einer intensiven Überprüfung der verwendeten Programme. Prinzipiell ließen sich Teile der Daten auch im Nachhinein in eine der verwendeten Strukturen bringen (unter Verwendung eines der Segmentationsprogramme oder von allen dreien im Vergleich), das wird jedoch als eine sehr aufwändige Anwendungsmöglichkeit eingeschätzt. Überlegenswert erscheint auch die ebenso aufwändige Möglichkeit, alte und neue Daten nach einer neuen (zu entwickelnden) Konvention in p-Formate zu bringen, die bessere Prognosemöglichkeiten bieten.

\subsection{Ermittlung der Kontaktsummen und Varianzen auf Basis der Nutzungswabrscheinlichkeiten (p-Werte)}

Auf Basis der p-Werte werden Kontaktsummen und Varianzen für Einzelsender und Senderkombinationen berechnet. Die Kontaktsumme beschreibt die Zahl der Kontakte, die ein Hörer mit dem betreffenden Werbeträger oder Werbemittel hat. Statistisch betrachtet handelt es sich um einen Erwartungswert, da dieser aus den Nutzungswahrscheinlichkeiten berechnet wird (siehe Unger et al. 2003: 68). In den folgenden Bereichen wurden Kontaktsummen als p-Werte für den Werbeträger- oder Werbemittelkontakt berechnet: Einzelsender pro durchschnittliche Stunde, Senderkombinationen pro durchschnittliche Stunde sowie für Einzelsender und Senderkombinationen pro Zeitabschnitt. Die Kontaktsumme K einer Senderkombination wird bei m Medienkontakteinheiten laut Codeplan MA 2000 EM II (ma_00_EM_II_cdb.pdf: 80) wie folgt berechnet: $\mathrm{K}=\mathrm{p} 1+\mathrm{p} 2+\ldots+\mathrm{pm}$. Kontaktsummen sind also nichts anderes als addierte oder auf durchschnittliche Zeitintervalle bezogene p-Werte (z. B. durchschnittliche Stunde: 08.00 bis $9.00 \mathrm{Uhr}$ ).

Ähnliches gilt für die Varianzen. Eine Varianz V ist gleich p1*(1-p1) und die Varianz einer Senderkombination besteht aus der Summe der Varianzen pro Einzelsender. Sie dienen als Maß für die Streuung innerhalb der Stichprobe und sollen quasi die Zielgenauigkeit bei der Mediaplanung beschreiben (Unger et al. 2003: 17).

Kontaktsummen und Varianzen von Medienkombinationen sowie die Nutzungswahrscheinlichkeiten für durchschnittliche Zeitintervalle werden laut Codeplan MA 2000 EM II (ma_00_EM_II_cdb.pdf: 80) immer nur für die so genannten „zulässigen“ Zeiten, also für die mit Werbung belegbaren Stunden, pro Individuum aus den Nutzungswahrscheinlichkeiten errechnet.

\subsection{Zusammenfassung: abgeleitete Variablen}

Gemeinsam haben die aufbereiteten Nutzungswahrscheinlichkeiten, dass jeweils pWerte mit Zahlen zwischen 0 und 100 berechnet wurden. Diese Daten könnten daher auch problemlos über die Zeit in eine gemeinsame Matrix eingespielt werden. Allerdings wurden bei der Segmentation drei unterschiedliche Programme verwendet, so dass eine Vergleichbarkeit kaum einzuschätzen ist. Die Problematik bezieht sich auch auf die aus den Nutzungswahrscheinlichkeiten gebildeten Kontaktsummen und Varianzen, die zudem nur für die „werberelevanten“ Zeiten berechnet wurden. Die Aggregationen (Zusammenfassungen) können jedoch problemlos übernommen werden. 


\section{Fazit}

Wie die Dokumentenanalyse gezeigt hat, lassen sich aus den Media-Analysen lange Zeitreihen erstellen, welche die Radiorezeption von 1976 an nachzeichnen können. Insbesondere die originären Senderrezeptionsdaten zu General- und Zeitfilter stehen dafür zur Verfügung und lassen - methodisch unbedenklich - über die Zeit ermitteln, in welchem Jahr welche Sender schon mal gehört wurden und wie weit die letzte Nutzung zurückliegt. Methodisch bedenklicher ist die longitudinale Nutzung der Frequenz- und der Tagesablaufvariablen einzuschätzen, da sie methodisch mit einem anderen Ansatz (Hörhäufigkeit pro Sender vs. Hörhäufigkeit pro Zeitabschnitt pro Sender) oder bezogen auf unterschiedliche Zeiteinheiten (Viertelstunden vs. [Halb-]Stunden) erhoben wurden. Allerdings können auch sie unter Beachtung der zu erwartenden Methodeneffekte für longitudinale Analysen genutzt werden. Ein Großteil der abgeleiteten Variablen kann jedoch erst nach einer genaueren Analyse der zur Ermittlung der p-Werte eingesetzten Segmentationsprogramme methodisch abgesichert über die Zeit genutzt werden. ${ }^{20}$

Ziel dieses Beitrags war es, die Möglichkeiten der Zeitreihenbildung mit Media-Analysen transparent und für andere nachvollziehbar darzustellen, auch um somit der Forderung von Lauf und Peiser (1999: 231ff.) nach einer ausführlichen Methodendokumentation bei der Aufbereitung von Datensätzen zu folgen. In einem nächsten Schritt können aus unterschiedlichsten Blickrichtungen Zeitreihen gebildet, statistisch geprüft und interpretiert werden. Für vertiefte Untersuchungen erscheint es sinnvoll, die Zeitreihen nach soziodemographischen Charakteristika differenziert zu erstellen: Beispielsweise können unter dem Oberbegriff Lebenszyklus Entwicklungen von Tendenzen bezüglich Alter, Kohorte, Geschlecht, Familienstand und Berufsstand untersucht werden. Unter dem Oberbegriff Sozialstatus lassen sich Entwicklungen bezüglich Bildung, Berufsstatus und Einkommen analysieren. ${ }^{21}$

Darüber hinaus sollten analoge Dokumentenanalysen die Fernseh-, Zeitschriftenund Zeitungsvariablen der Media- und Leser-Analyse zum Untersuchungsgegenstand haben, da diese ein ähnliches Potenzial für die longitudinale Analyse aufweisen.

\section{Literatur}

AG.MA, 2004. AG.MA-Aufnahme-, Erhebungs, Ausweisungs- und Finanzierungsrichtlinien. AG.MA: Frankfurt am Main. http://www.agma-mmc.de/01_profil/Aufnahme-Richtlinien_ Stand251104.pdf, Stand: 25. November 2004.

Akinci, H., 2004. Leitfaden für den Umgang mit Tagesablaufdaten. http://www.wiso.uni-koeln. $\mathrm{de} /$ medien/download/tagesablaufdokumentation.pdf, erstellt am 28.06.04.

Akinci, H., 2006. Lebensstile, Sozialstatus und Mediennutzung. In Hagenah, J. \& Meulemann, H. (Hrsg.). Sozialer Wandel und Mediennutzung in der Bundesrepublik Deutschland. (S. 230-246). Münster: LIT Verlag.

Behrens, K. \& Löffler, U., 1999. Aufbau des ADM-Stichproben.Systems. In ADM Arbeitskreis Deutscher Markt- und Sozialforschungsinstitute e.V.; AG.MA Arbeitsgemeinschaft Media-

20 Die Nutzungswahrscheinlichkeiten innerhalb eines einzelnen Querschnittes können jedoch unproblematisch genutzt werden.

21 Geprüft wird zudem, inwieweit Entwicklungen in Abhängigkeit vom Lebensstil bspw. unter der Verwendung der Operationalisierungsstrategie von Wahl $(2006 ; 2003 ; 1997)$ untersucht werden können. 
Analyse e.V. (Hrsg.). Stichprobenverfahren in der Umfrageforschung. Opladen: Leske + Budrich

Berg, K. \& Kiefer, M. L., 1992. Massenkommunikation IV. Eine Langzeitstudie zur Mediennutzung und Medienbewertung 1964-1990. Baden-Baden: Nomos Verlagsgesellschaft.

Berg, K. \& Ridder, C. M., 2002. Massenkommunikation VI. Eine Langzeitstudie zur Mediennutzung und Medienbewertung 1964-2000, Baden-Baden: Nomos Verlagsgesellschaft.

Best, H. \& Hagenah, J., 2006. Vom persönlichen zum telefonischen Interview: Probleme der Stichproben-Zusammensetzung und des Antwortverhaltens. In Hagenah, J. \& Meulemann, H. (Hrsg.). Sozialer Wandel und Mediennutzung in der Bundesrepublik Deutschland: Nutzung der Daten der Media-Analyse für Sekundüranalysen. (S. 35 - 56). Münster: LIT Verlag.

Buß, M., 1998. Leistungsfähigkeit und Grenzen der Media Analyse. In Lindner-Braun, C. (Hrsg.). Radioforschung - Konzepte, Instrumente und Ergebnisse aus der Praxis. (S. 77-82) Opladen: Westdeutscher Verlag.

Doh, M. \& Kaspar, R., 2006. Entwicklung und Determinanten der Internetdiffusion bei älteren Menschen. In Hagenah, J. \& Meulemann, H. (Hrsg.). Sozialer Wandel und Mediennutzung in der Bundesrepublik Deutschland. (S. 139-156). Münster: LIT Verlag.

Ehrenberg, M., 2005. Mittagessen mit Moderator. Zum Zusammenhang von Zeitbudget, Tagesablauf und Parallelnutzung. Unveröffentlichte Magisterarbeit: Friedrich-Schiller-Universität Jena. http://www.wiso.uni-koeln.de/medien/download/Mittagessen_mit_Moderator-Maria_Ehrenberg_Maerz_2006_1.pdf

Engel, B. \& Best, S., 2001. Mediennutzung und Medienbewertung im Kohortenvergleich. In Media Perspektiven 11/ 2001, 554-563.

Ernst, O., 1995. Anmerkungen zu Informationsbestand und Funktion der Media.Analyse. Vortrag gehalten auf der Mitgliederversammlung der AG.MA 1978. In Scheler, H. E. (Hrsg.). Dr. Otmar Ernst - Ausgewählte Reden - Ein Leitfaden durch 40 Jabre Mediaforschung. (S. 87-96) Frankfurt am Main: AG.MA.

Fachinger, U., 2006. Nutzung der Daten der Media-Analyse zur Identifizierung von altersspezifischem Ausgabeverhalten. In Hagenah, J. \& Meulemann, H. (Hrsg.). Sozialer Wandel und Mediennutzung in der Bundesrepublik Deutschland. (S. 157-173). Münster: LIT Verlag.

Frisch, A.-L., 2005. Senderbindung im Hörfunk. Eine Sekundärauswertung der Media-Analyse 2000 zur Hörfunknutzung in Deutschland. Magisterarbeit: Friedrich-Schiller-Universität Jena. http://www.wiso.uni-koeln.de/medien/download/Publikationen/Magisterarbeit-SenderbindungimHoerfunk.pdf

Gabler, S. \& Häder, S., 1997. Überlegungen zu einem Stichprobendesign für Telefonumfragen in Deutschland. In: ZUMA-Nachrichten, 41, 1997, S. 7-18.

Gonser, N. \& Scherer, H., 2006. Die vielfältige Mediennutzung älterer Menschen: Eine Auswertung der Media-Analysen im Hinblick auf Menschen ab 50 Jahren. In Hagenah, J. \& Meulemann, H. (Hrsg.). Sozialer Wandel und Mediennutzung in der Bundesrepublik Deutschland. (S. 122-138). Münster: LIT Verlag.

Hagenah, J., 2003a. ma-Analyse-Leitfaden. http://www.wiso.uni-koeln.de/medien/download/ ma_analyse_leitfaden.pdf, erstellt am 19.09.2003.

Hagenah, J., 2003b. ma-Beispielanalyse „Sportaktivität“. http://www.wiso.uni-koeln.de/medien/ download/ma_analysebeispiel_sportaktivitaet.pdf, erstellt am 19.09.2003.

Hagenah, J., 2004a. Leitfaden für eine individuelle Aufbereitung der ma-Daten. http://www. wiso.uni-koeln.de/medien/download/leitfaden_ma_daten_aufbereitung_mlfz.pdf, erstellt am 07.04.2004.

Hagenah, J., 2004b. Sozialer Wandel und Mediennutzung in der Bundesrepublik Deutschland: Nutzung der Daten der Media-Analyse von 1972 bis 2000 für Sekundäranalysen. Tagungsbericht zum 1. Workshop des Medienwissenschaftlichen Lehr- und Forschungszentrums (MLFZ) am 05./06. November in Köln. ZA-Information 55, S. 137-141.

Hagenah, J. \& Akinci, H., 2003a. Zwischenbericht über die Arbeit des medienwissenschaftlichen Lehr- und Forschungszentrums. Unveröffentlichter Arbeitsbericht: Universität zu Köln. [Einige Auszüge sind veröffentlicht: http://www.wiso.uni-koeln.de/medien erstellt am 24.09.2003.]

Hagenah, J. \& Akinci, H., 2003b. Sekundäranalysen der Daten der Media-Analyse mit Hilfe des 
Serviceangebots des Medienwissenschaftlichen Lehr- und Forschungszentrums (MLFZ). ZAInformation 53, 182-190.

Hagenah, J. \& Best, H., 2005. Die Rolle von Auswahl- und Befragungsverfahren am Beispiel der Media-Analyse. Grundgesamtheit und Inhalte im Vergleich zwischen telefonisch und persönlich-mündlich erhobenen Daten. In Gehrau, V., Fretwurst, B., Krause, B. \& Daschmann, G. (Hrsg.). Auswablverfahren in der Kommunikationswissenschaft. (S. 223-250). Köln: Herbert von Halem Verlag.

Hagenah, J. \& Meulemann, H., 2006. Sozialer Wandel und Mediennutzung in der Bundesrepublik Deutschland: Nutzung der Daten der Media-Analyse für Sekundäranalysen: In Hagenah, J. \& Meulemann, H. (Hrsg.). Sozialer Wandel und Mediennutzung in der Bundesrepublik Deutschland. (S. 7-16). Münster: LIT Verlag.

Hagenah, J., Meulemann, H. \& Akinci, H., 2006. European Data Watch: German Media-Analyse (MA): A large scale commercial data source available for secondary analyses on media use and social change. Schmollers Jabrbuch. Journal of Applied Social Science Studies / Zeitschrift für Wirtschafts- und Sozialwissenschaften, 1, 73-83.

Hagenah, J. \& Schliermann, R. 2006. Fußball für Alle: Binden Welt- und Europameisterschaften ein größeres Publikum an die Bundesliga?: In Hagenah, J. \& Meulemann, H. (Hrsg.). Sozialer Wandel und Mediennutzung in der Bundesrepublik Deutschland. (S. 93-119). Münster: LIT Verlag.

Hasebrink, U., 2002. Status Quo und Perspektiven internationaler akademischer Nutzungsforschung. Hamburg: Hans-Bredow-Institut.

Hoffmann, H. \& Müller, D. K., 2003. Gelungener Umstieg von Face-to-Face zu CATI: Zuverlässiges Erhebungsinstrument zur Ermittlung der Radionutzung. In Arbeitsgemeinschaft MediaAnalyse e.V. (Hrsg.). Dokumentation der Experimente und Methoden zur Optimierung der media-analyse-Radio. (S. 11-32). Frankfurt am Main: AG.MA.

Hoffmeyer-Zlotnik, J.H.P., 1997. Random-Route-Stichproben nach ADM. In Gabler, S. \& Hoffmeyer-Zlotnik, J.H.P. (Hrsg.). Stichproben in der Umfragepraxis. (S. 33-42). Opladen: Westdeutscher Verlag.

Kaase, M. \& Langenbucher, W. R., 1986. Medienwirkungen auf Gesellschaft und Politik. In: Deutsche Forschungsgemeinschaft (Hrsg.). Medienwirkungsforschung in der Bundesrepublik Deutschland. Teil I: Berichte und Empfehlungen. Weinheim: VCH-Verlag. 13-28.

Kiefer, M. L., 1998. Ein Unikat in der Rezeptionsforschung: Langzeitstudie Massenkommunikation zur Mediennutzung und Medienbewertung. In Klingler, W., Roters, G. \& Zöllner, O. (Hrsg.). Fernsehforschung in Deutschland: Themen - Akteure - Methoden: Band 1. Baden-Baden: Nomos Verlagsgesellschaft. 17-29.

Kiefer, M. L., 1999. Wie betreibt man wissenschaftliche Langzeitforschung. Eine Replik auf die Kritik von Lauf/ Peiser. In: Rundfunk und Fernsehen, 47/ 2, 243-256.

Koschnick, W. J., 2004a. Werbemittelkontakt (Werbemittelkontaktchance). In Koschnick, W. J. (Hrsg.). Focus-Lexikon Werbeplanung - Mediaplanung - Marktforschung - Kommunikationsforschung Mediaforschung. www.medialine.-focus.de/PM1D/PM1DB/PM1DBF/pm1dbf. htm?snr=6067, gelesen am 30.07.2004.

Koschnick, W. J., 2004b. Abfrage (Medienabfrage). In Koschnick, W. J. (Hrsg.). Focus-Lexikon Werbeplanung - Mediaplanung - Marktforschung - Kommunikationsforschung - Mediaforschung. www.medialine.focus.de/PM1D/PM1DB/PM1DBF/pm1dbf.htm?stichwort=abfrage $\& \mathrm{x}=0 \& \mathrm{y}=0$, gelesen am 15.08.2004.

Koschnick, W. J., 2004c. Segmentation (Segmentanalyse). In Koschnick, W. J. (Hrsg.). FocusLexikon Werbeplanung - Mediaplanung - Marktforschung - Kommunikationsforschung Mediaforschung. www.medialine.focus.de/PM1D/PM1DB/PM1DBF/pm1dbf.htm?stichwort =Segmentation, gelesen am 17.11.2004.

Koschnick, W. J., 2004d. Filterfrage (Filterführung, Screening). In Koschnick, W. J. (Hrsg.). Focus-Lexikon Werbeplanung - Mediaplanung- Marktforschung - Kommunikationsforschung - Mediaforschung. http://www.medialine.focus.de/PM1D/PM1DB/PM1DBF/pm1dbf.htm? stichwort $=$ Filterfrage $+\& x=0 \& y=0$, gelesen am 18.11.2004.

Kubitschke, L. \& Trebbe, J., 1992. Zur Ermittlung einer medienübergreifenden Nutzungstypo- 
logie. Eine explorative Sekundäranalyse der Media-Analyse 1988. In: Media Perspektiven, 3, 199-212.

Lauf, E., 1999. Primär sekundäranalysiert: Tageszeitungsnutzung in der Media-Analyse, der Allensbacher Werbeträger Analyse und in der Langzeitstudie Massenkommunikation. www.dgpuk. de/fg_meth/abs14.htm, gelesen am 12.02.2005.

Lauf, E. \& Peiser, W., 1999. Validität der Studie Massenkommunikation. In: Rundfunk und Fernsehen, 47/2, 231-242.

Lauf, E., 2006. Tageszeitungsnutzung in der Media-Analyse, der Allensbacher Werbeträgeranalyse und in der Langzeitstudie Massenkommunikation. In Hagenah, J. \& Meulemann, H. (Hrsg.). Sozialer Wandel und Mediennutzung in der Bundesrepublik Deutschland. (S. 57-70). Münster: LIT Verlag.

MA 75 Berichtsband, 1975. Vorwort. In Arbeitsgemeinschaft Media-Analyse e.V. (Hrsg.). MediaAnalyse 1975. (S. 4). Frankfurt/M.: Media-Micro-Census GmbH

Mai, L., 2003. Die Media-Analyse Radio. Eine Methodenbeschreibung. Frankfurt: ARD-Werbung SALES \& SERVIES GmbH Corporate Communication.

Meulemann, H., Hagenah, J. \& Akinci, H. 2005. Die Media-Analysen. Synopse des Datenbestands und Nutzungschancen für Sekundäranalysen des sozialen Wandels in Deutschland seit 1954. In: König, C., Stahl, M. \& Wiegand, E. (Hrsg.). Datenfusion und Datenintegration. 6. Wissenschaftliche Tagung im Auftrag ADM Arbeitskreis Deutscher Markt- und Sozialforschungsinstitute, Arbeitsgemeinschaft Sozialwissenschaftlicher Institute e.V. (ASI) und Statistisches Bundesamt. (S. 54-69) Bonn: Informationszentrum Sozialwissenschaften.

Müller, D. K. \& Mai, L., 2006. Das Erhebungsmodell der Media-Analyse Radio. In Hagenah, J. \& Meulemann, H. (Hrsg.). Sozialer Wandel und Mediennutzung in der Bundesrepublik Deutschland: Nutzung der Daten der Media-Analyse für Sekundäranalysen. (S. 18-34). Münster: LIT Verlag.

Murray, J. P.,1991. Nothing Lasts Forever: Instability in Longitudinal Studies of Media and Society. In: Anderson, J. (Hrsg.). Communication Yearbook 14. Newbury Park. 102-110.

Peiser, W., 1996. Die Fernsehgeneration. Opladen: Westdeutscher Verlag.

Ridder, C. M. \& Engel, B., 2005. Massenkommunikation 2005: Images und Funktionen der Massenmedien im Vergleich. In: Media Perspektiven 9/2005, 422-449.

Risel, M., 2006. Westdeutsche Lebensstile Ende des 20. Jahrhunderts. Eine empirische Untersuchung zum Zusammenhang von Sozialstruktur und Lebensstil. In Hagenah, J. \& Meulemann, H. (Hrsg.). Sozialer Wandel und Mediennutzung in der Bundesrepublik Deutschland. (S. 205229). Münster: LIT Verlag.

Risel, M., 2005: Westdeutsche Lebensstile Ende des 20. Jabrhunderts. Eine empirische Untersuchung zum Zusammenhang von Sozialstruktur und Lebensstil. Universität Tübingen: unveröffentlichte Magisterarbeit. http://www.wiso.uni-koeln.de/medien/download/MA_Risel_Lebensstile.pdf

Scheler, H.-E., 1979. Modalitäten der MA-Berichterstattung - Umfang, Termine, Möglichkeiten. $Z V+Z V, 36,1369-1373$

Scheler, H.-E., 1983. Experimente, ihr Anlaß und ihr Ergebnis. Vortrag auf der AG.MA-Mitgliederversammlung am 23. November 1983. In Arbeitsgemeinschaft Media-Analyse (Hrsg.) Dokumentation der Experimente 196 - 1983 zur Veränderung des Fragebogens der MA AG.MA Schriften Band 10, 387-393. Frankfurt: AG.MA.

Schönbach, K., Lauf, E., Stürzebecher, D. \& Peiser, W., 1997. Faktoren des Zeitungserfolgs. In Schönbach, K. (Hrsg.). Zeitungen in den Neunzigern: Faktoren ibres Erfolgs. 350 Tageszeitungen auf dem Prïfstand. (S. 61-112). Bonn: ZV Zeitungsverlag Service GmbH.

Seufert, W. \& Suckfüll, M., 2006. Zeitverfügbarkeit und Zeitbewertung als Erklärungsfaktoren der individuellen Mediennutzung. In Hagenah, J. \& Meulemann, H. (Hrsg.). Sozialer Wandel und Mediennutzung in der Bundesrepublik Deutschland. (S. 72-92). Münster: LIT Verlag.

Unger, F., Durante, N. V., Gabrys, E., Koch, R. \& Wailersbacher, R., 2003. Mediaplanung. Methodische Grundlagen und praktische Anwendungen. Berlin, Heidelberg, New York: SpringerVerlag.

Van Eimeren, B. \& Ridder, C.-M., 2001. Trends in der Nutzung und Bewertung der Medien 1970 bis 2000. In: Media Perspektiven, 11/2001. 538-553. 
Wahl, A., 1997: Strukturierte Pluralität. Lebensstile zwischen vertikalen Strukturbedingungen und intervenierenden Faktoren, Frankfurt a. M./ Berlin u.a.: Peter Lang.

Wahl, A., 2003. Veränderung von Lebensstilen. Frankfurt a.M.: Campus Verlag GmbH.

Wahl, A., 2006. Lebensstile im Kontext von Generationen- und Lebenszykluseinflüssen. In Hagenah, J. \& Meulemann, H. (Hrsg.). Sozialer Wandel und Mediennutzung in der Bundesrepublik Deutschland: Nutzung der Daten der Media-Analyse für Sekundäranalysen. (S. 175 - 204). Münster: LIT Verlag.

Weiß, R. \& Hasebrink, U., 1995. Hörertypen und ibr Medienalltag. Eine Sekundärauswertung der Media-Analyse 94 zur Radiokultur in Hamburg. Berlin: Vistas Verlag GmbH.

Weiß, R. \& Hasebrink, U., 1997. Hörertypen und ihr Medienalltag. Plädoyer für eine hörerzentrierte Nutzungsanalyse. In: Publizistik, 42/2, 164-180.

www.agma-mmc.de 2005. CASI-Methode. Frankfurt am Main: Arbeitsgemeinschaft Media-Analyse e.V. http://www.agma-mmc.de, gelesen am 18.10.2005.

www.rms.de 2004a. Radio Marketing Service RMS: Grundlagen der Radio-Planung III, gelesen am 18.11.2004.

www.rms.de 2004b. rms-Medialexikon: Werbemittelkontaktchance (WMKC), gelesen am 25.11.2004.

www.topradio.de 2004. Werbeträgerkontakt gelesen am 18.11.2004.

MA-Fragebögen und Codepläne befinden sich zum Download auf der MLFZ-Homepage (Fragebögen) bzw. können dort bestellt werden (Codepläne): www.wiso.uni-koeln.de/medien/

Fragebogen ma 1975 (1975). Fragebogen und Befragungsunterlagen MA 1975. Frankfurt (Main): Arbeitsgemeinschaft Media-Analyse e.V. und Media-Micro-Census GmbH. (ma_75_frb.pdf), erstellt am 14.05.04.

Fragebogen ma 1985 (1985). Befragungsunterlagen MA 1985. Frankfurt (Main): Arbeitsgemeinschaft Media-Analyse e.V. und Media-Micro-Census GmbH. (ma_85_frb.pdf), erstellt am 14.05.04.

Fragebogen ma 1999 EM (1999). MA 99 Radio. Frankfurt (Main): Arbeitsgemeinschaft Media-Analyse e.V. und Media-Micro-Census GmbH. (ma_99_EM_frb.pdf), erstellt am 14.05.04.

Fragebogen ma 2000 EM II (2000). MA 2000 Radio. CATI-Frageprogramm. 2. Welle. Frankfurt (Main): Arbeitsgemeinschaft Media-Analyse e.V. und Media-Micro-Census GmbH. (ma_00_ EM_II_frb.pdf), erstellt am 14.05.04.

Codeplan ma 1976 (1976). Media Analyse (MA 76). Frankfurt (Main): Arbeitsgemeinschaft MediaAnalyse e.V. und Media-Micro-Census GmbH. Archiv des Medienwissenschaftlichen Lehrund Forschungszentrums: ma_76_cdb.pdf, erstellt am 14.11.03.

Codeplan ma 1985 (1985). MA 85. Datensatz Codeplan. Frankfurt (Main): Arbeitsgemeinschaft Media-Analyse e.V. und Media-Micro-Census GmbH. Archiv des Medienwissenschaftlichen Lehr- und Forschungszentrums: ma_85_cdb.pdf, erstellt am 14.11.03.

Codeplan ma 1999 EM (1999). MA 99 Radio. Datensatz Codeplan. Frankfurt (Main): Arbeitsgemeinschaft Media-Analyse e.V. und Media-Micro-Census GmbH. Archiv des Medienwissenschaftlichen Lehr- und Forschungszentrums: ma_99_EM_cdb.pdf, erstellt am 14.11.03. 